\title{
Robust Hierarchical Adaptive Fuzzy Relative Motion Coordination for Feature Points of Two Rigid Bodies with Input and Output Constraints
}

\author{
Liang Sun, Member, IEEE and Jingjing Jiang, Member, IEEE
}

\begin{abstract}
A model-based six-degrees-of-freedom relative motion coordinated control approach is developed for the relative position tracking and attitude synchronization between feature points of two rigid bodies subject to control input constraints, output constraints and model uncertainties. In the designing framework of adaptive backstepping control technique, the control input saturation is compensated by the nonlinear antiwindup compensator and the output constraints are handled by the barrier Lyapunov function-based backstepping design. The unknown misalignment vector of the feature point with respect to the center of the mass for the chaser is estimated by the element-wise adaptive law, while the model uncertainties and unknown dynamical couplings are compensated by the adaptive hierarchical fuzzy logic system to decrease the computational burden with respect to the traditional adaptive fuzzy system. The ultimately uniformly bounded convergence of the relative pose and relative velocities is analyzed in the Lyapunov framework and the effectiveness of the proposed approach is validated by the numerical simulations.
\end{abstract}

Index Terms-Rigid bodies, feature points, pose coordination, adaptive control, hierarchical fuzzy system, control input saturation, output constraints.

\section{NOMENCLATURE}

$\mathcal{F}_{o} \quad$ Inertial frame

$\mathcal{F}_{c} \quad$ Chaser feature-point-fixed frame

$\mathcal{F}_{t} \quad$ Target feature-point-fixed frame

$\mathrm{O} \quad$ Origin of $\mathcal{F}_{o}$

P Origin of $\mathcal{F}_{t}$

Q Origin of $\mathcal{F}_{c}$

C Mass center of chaser

$\mathbf{T} \quad$ Mass center of target

$\boldsymbol{l} \quad$ Position of $\mathbf{Q}$ with respect to $\mathbf{C}, \mathrm{m}$

$\boldsymbol{l}_{t} \quad$ Position of $\mathbf{P}$ with respect to $\mathbf{T}, \mathrm{m}$

$\boldsymbol{r} \quad$ Position of $\mathbf{Q}$ with respect to $\mathbf{O}, \mathrm{m}$

$\boldsymbol{r}_{t} \quad$ Position of $\mathbf{P}$ with respect to $\mathbf{O}, \mathrm{m}$

$\boldsymbol{r}_{e} \quad$ Position of $\mathbf{Q}$ with respect to $\mathbf{P}, \mathrm{m}$

This work was supported by the National Natural Science Foundation of China (No. 61903025), the China Scholarship Council (No. 201906465028) and the Fundamental Research Funds for the Central Universities (Nos. FRFBD-19-002A, FRF-GF-18-0028B).

L. Sun is with the Key Laboratory of Knowledge Automation for Industrial Processes of Ministry of Education, School of Automation and Electrical Engineering, University of Science and Technology Beijing, Beijing 100083, China, and with the Institute of Artificial Intelligence, University of Science and Technology Beijing, Beijing 100083, China. (e-mail: liangsundustb.edu.cn)

J. Jiang is with the Department of Aeronautical and Automotive Engineering, Loughborough University, Leicestershire, England LE11 3TU, United Kingdom.(e-mail: J.Jiang2@ lboro.ac.uk)

\section{INTRODUCTION}

$\mathbf{T}$ HE pose motion control of the rigid body is a hot topic in the automation field for many years, since it is the physical nature of many kinds of man-made moving bodies in practice, such as the motions of automotive vehicles [1], aircrafts [2], spacecraft [3], marine vessels [4] and formations [5]. In particular, the relative motion between two vehicles is very common in advanced modern industry, such as the air refueling [6], shipboard landing [7], space docking [8] and underwater docking [9]. In these missions, the close-range relative pose motion model should be established carefully for the model-based control system design. Specifically, the feature points on the surfaces of these moving bodies, such as the positions of refueling pipe, landing points, and docking ports, should be considered in the relative pose modeling 
and control. The feature points of two vehicles are generally misaligned with their centers of mass, while the size and shape of the rigid bodies cannot be ignored in the advanced missions. Thus, the relative translational-rotational control design between the feature points of two rigid bodies should be considered for these high-precision control systems.

The linear control method is apparently unsuitable for the relative dynamics between two feature points of the rigid bodies, since the relative translational motion is generally coupled with the relative rotational motion. Moreover, the classical linear controllers, including transfer function-based control, classical proportional-integral-differential control and linear quadratic regulation control, cannot deal with multiple input multiple output problems with strong couplings in the relative pose dynamics. To this end, many effective nonlinear control methods were developed for the pose control of a single rigid body [10], the relative pose control of two rigid bodies [11], and the formation control of multiple rigid bodies [12] under the fully-actuated and under-actuated cases. Regardless of the relative pose control for these rigid bodies, the model uncertainties are one of the significant problems in the control systems. Meanwhile, many effective control designing methods including adaptive control [13][14] and intelligent control [15] were also applied to deal with the unknown constants and functions in the dynamics. As an effective and representative control technique, the model-based adaptive hierarchical fuzzy logic control approach is a very important method to deal with the unknown nonlinear function in dynamical systems [16][17], due to its stronger ability of the online approximation in the prescribed compact set and smaller computational burden [18]. In particular, the modelbased hierarchical fuzzy logic control method is very suitable for the practical rigid-bodies systems, such as robots [19][20], helicopters [21], spacecraft [22][23] and other nonlinear systems [24], since the dynamical model of rigid bodies could be established explicitly for improving the control precision in practice. Besides the model uncertainties, the input and output constraints are also the significant problems in control systems, since the constraints are generally related with the stability, safety and reliability of the systems [25]. Because of the constraints of the actuator outputs for practical systems, the exaggerate control commands cannot be permitted and the stability of the controlled system cannot be guaranteed [26][27]. Moreover, the output constraints are generally resulted from the bounded requirements of partial states to avoid the computational singularity [28] or satisfy the prescribed performances [29]. The input and output constraints have been considered in many systems, such as path following of autonomous underwater vehicles [30], final phase proximity operations of spacecraft rendezvous [31], linear mechanical systems [32] and nonlinear dynamical systems [33].

Based on the observations of previous studies on adaptive hierarchical fuzzy control for the rigid bodies subject to input and output constraints, this study focuses on the modelbased relative position tracking and attitude synchronization control system design for feature points of two fully-actuated rigid bodies named chaser and target, where the six-degreesof-freedom relative motion model of two feature points is established based on the classical Newton-Euler method, while the model uncertainties and constraints are considered in the control design. The main features of this study are given as the following three aspects. Firstly, the relative pose dynamics are modeled in the feature-point-fixed frame of the active chaser, and the feature-point pose of the passive target is also involved in the relative motion model to improve the modelling precision. In particular, the relative attitude is described by the modified Rodrigues parameters [34] to avoid the defects of multiple equilibriums in rotational matrix description, the singularity in Euler-angles description and the parameter redundancy in quaternion description. Secondly, the adaptive hierarchical fuzzy logic control approach is combined with the robust adaptive backstepping technique for the integrated six-degrees-of-freedom relative pose control design, where the unknown constant misalignment vector is estimated by the element-wise adaptive laws, and the unknown bounded nonlinear function, including model uncertainties and dynamical couplings, is approximated by the adaptive hierarchical fuzzy logic system with assistance of a switching functionbased robust adaptive strategy. Lastly, the input and output constraints are handled by the anti-windup compensator and the barrier Lyapunov function-based controller, while the ultimately uniformly bounded stability of the controlled system is derived in a Lyapunov framework. Simulation results validate the effectiveness and advantages of the proposed modelling and control design approaches.

The rest of the paper is divided into four sections. Section II presents the mathematical description and the control objectives for relative motion coordination. Section III provides the procedure of relative motion controller design and analyzes the stability of the closed-loop system. Numerical simulations are conducted in Section IV to support the theoretic developments. Finally, conclusions and future works are drawn in Section V.

\section{Problem Statement}

Consider the feature points of a rigid body, named active chaser, tracking the feature points of another rigid body, named passive target, and synchronizing the attitude of the chaser with respect to the target in the three-dimensional space. This scenario is shown in Figure 1 , where $\mathcal{F}_{o}=\left\{O x_{i} y_{i} z_{i}\right\}, \mathcal{F}_{t}=$ $\left\{P x_{t} y_{t} z_{t}\right\}$, and $\mathcal{F}_{c}=\{Q x y z\}$.

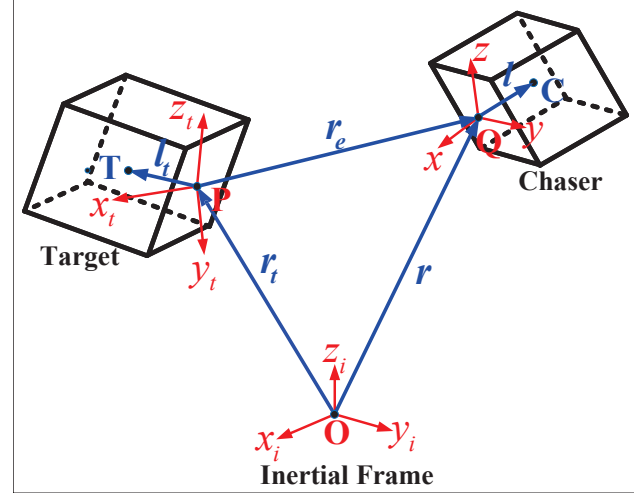

Fig. 1. Scenario of relative pose motion between feature points. 
The chaser's pose kinematics and dynamics represented in frame $\mathcal{F}_{c}$ are modeled as

$$
\left\{\begin{array}{l}
\dot{\boldsymbol{p}}=\mathcal{A} \boldsymbol{p}+\mathcal{B} \boldsymbol{q} \\
\mathcal{M} \dot{\boldsymbol{q}}+\mathcal{C} \boldsymbol{q}=\left(I_{6}+\mathcal{E}\right)(\boldsymbol{u}+\boldsymbol{d})
\end{array}\right.
$$

where $\boldsymbol{p}=\left[\boldsymbol{r}^{T}, \boldsymbol{\sigma}^{T}\right]^{T} ; \boldsymbol{q}=\left[\boldsymbol{v}^{T}, \boldsymbol{\omega}^{T}\right]^{T} ; \mathcal{A}=$ $\operatorname{diag}\left\{-S(\boldsymbol{\omega}), O_{3}\right\} ; \mathcal{B}=\operatorname{diag}\left\{I_{3}, G(\boldsymbol{\sigma})\right\} ; G(\boldsymbol{\sigma})=\frac{1}{4}[(1-$ $\left.\left.\boldsymbol{\sigma}^{T} \boldsymbol{\sigma}\right) I_{3}+2 S(\boldsymbol{\sigma})+2 \boldsymbol{\sigma} \boldsymbol{\sigma}^{T}\right]$

$$
\begin{gathered}
\mathcal{M}=\left[\begin{array}{cc}
m I_{3} & -m S(\boldsymbol{l}) \\
m S(\boldsymbol{l}) & J-m S^{2}(\boldsymbol{l})
\end{array}\right] ; \quad \mathcal{E}=\left[\begin{array}{cc}
O_{3} & O_{3} \\
S(\boldsymbol{l}) & O_{3}
\end{array}\right] ; \\
\mathcal{C}=\left[\begin{array}{cc}
m S(\boldsymbol{\omega}) & -m S(\boldsymbol{\omega}) S(\boldsymbol{l}) \\
m S(\boldsymbol{l}) S(\boldsymbol{\omega}) & S(\boldsymbol{\omega}) J-m S(\boldsymbol{\omega}) S^{2}(\boldsymbol{l})
\end{array}\right] ;
\end{gathered}
$$

$\boldsymbol{u}=\left[\boldsymbol{f}^{T}, \boldsymbol{\tau}^{T}\right]^{T} ; \boldsymbol{d}=\left[\boldsymbol{w}^{T}, \boldsymbol{\delta}^{T}\right]^{T}$. In particular, due to the unsymmetrically saturated outputs of the actuators mounted on the controlled chaser, the control input $\boldsymbol{u}=$ $\left[u_{1}, u_{2}, u_{3}, u_{4}, u_{5}, u_{6}\right]^{T}$ in (1) is constrained by

$$
-u_{k_{\min }} \leq u_{k} \leq u_{k_{\max }}, k=1,2, \cdots, 6
$$

Thus the control input $u_{k}(k=1,2, \cdots, 6)$ can be rewritten by

$$
u_{k}= \begin{cases}u_{k_{\max }} & \text { if } u_{k_{0}}>u_{k_{\max }} \\ u_{k_{0}} & \text { if }-u_{k_{\min }} \leq u_{k_{0}} \leq u_{k_{\max }} \\ -u_{k_{\min }} & \text { if } u_{k_{0}}<-u_{k_{\min }}\end{cases}
$$

where $\boldsymbol{u}_{0}=\left[u_{1_{0}}, u_{2_{0}}, u_{3_{0}}, u_{4_{0}}, u_{5_{0}}, u_{6_{0}}\right]^{\mathrm{T}}$ is the control command to be designed.

The tumbling target's pose kinematics and dynamics are established in frame $\mathcal{F}_{t}$ as

$$
\left\{\begin{array}{l}
\dot{p}_{t}=\mathcal{A}_{t} \boldsymbol{p}_{t}+\mathcal{B}_{t} \boldsymbol{q}_{t} \\
\mathcal{M}_{t} \dot{\boldsymbol{q}}_{t}+\mathcal{C}_{t} \boldsymbol{q}_{t}=\mathcal{E}_{t} \boldsymbol{d}_{t}
\end{array}\right.
$$

where $\boldsymbol{p}_{t}=\left[\boldsymbol{r}_{t}^{T}, \boldsymbol{\sigma}_{t}^{T}\right]^{T} ; \boldsymbol{q}_{t}=\left[\boldsymbol{v}_{t}^{T}, \boldsymbol{\omega}_{t}^{T}\right]^{T} ; \mathcal{A}_{t}=$ $\operatorname{diag}\left\{-S\left(\boldsymbol{\omega}_{t}\right), O_{3}\right\} ; \mathcal{B}_{t}=\operatorname{diag}\left\{I_{3}, G\left(\boldsymbol{\sigma}_{t}\right)\right\} ; \boldsymbol{d}_{t}=\left[\boldsymbol{w}_{t}^{T}, \boldsymbol{\delta}_{t}^{T}\right]^{T} ;$

$$
\begin{aligned}
\mathcal{M}_{t} & =\left[\begin{array}{cc}
m_{t} I_{3} & -m_{t} S\left(\boldsymbol{l}_{t}\right) \\
m_{t} S\left(\boldsymbol{l}_{t}\right) & J_{t}-m_{t} S^{2}\left(\boldsymbol{l}_{t}\right)
\end{array}\right] ; \mathcal{E}_{t}=\left[\begin{array}{cc}
I_{3} & O_{3} \\
S\left(\boldsymbol{l}_{t}\right) & I_{3}
\end{array}\right] ; \\
\mathcal{C}_{t} & =\left[\begin{array}{cc}
m_{t} S\left(\boldsymbol{\omega}_{t}\right) & -m_{t} S\left(\boldsymbol{\omega}_{t}\right) S\left(\boldsymbol{l}_{t}\right) \\
m_{t} S\left(\boldsymbol{l}_{t}\right) S\left(\boldsymbol{\omega}_{t}\right) & S\left(\boldsymbol{\omega}_{t}\right) J_{t}-m_{t} S\left(\boldsymbol{\omega}_{t}\right) S^{2}\left(\boldsymbol{l}_{t}\right)
\end{array}\right] .
\end{aligned}
$$

Define the relative pose and velocity between $\mathbf{Q}$ and $\mathbf{P}$ in frame $\mathcal{F}_{c}$ as

$$
\left\{\begin{array}{l}
\boldsymbol{r}_{e}=\boldsymbol{r}-R \boldsymbol{r}_{t} \\
\boldsymbol{\sigma}_{e}=\boldsymbol{\sigma} \otimes \boldsymbol{\sigma}_{t}^{-1} \\
\boldsymbol{v}_{e}=\boldsymbol{v}-R \boldsymbol{v}_{t} \\
\boldsymbol{\omega}_{e}=\boldsymbol{\omega}-R \boldsymbol{\omega}_{t}
\end{array}\right.
$$

where

$$
\boldsymbol{\sigma} \otimes \boldsymbol{\sigma}_{t}^{-1}=\frac{\boldsymbol{\sigma}_{t}\left(\boldsymbol{\sigma}^{T} \boldsymbol{\sigma}-1\right)+\boldsymbol{\sigma}\left(1-\boldsymbol{\sigma}_{t}^{T} \boldsymbol{\sigma}_{t}\right)-2 S\left(\boldsymbol{\sigma}_{t}\right) \boldsymbol{\sigma}}{1+\boldsymbol{\sigma}_{t}^{T} \boldsymbol{\sigma}_{t} \boldsymbol{\sigma}^{T} \boldsymbol{\sigma}+2 \boldsymbol{\sigma}_{t}^{T} \boldsymbol{\sigma}}
$$

and

$$
R=I_{3}-\frac{4\left(1-\boldsymbol{\sigma}_{e}^{T} \boldsymbol{\sigma}_{e}\right)}{\left(1+\boldsymbol{\sigma}_{e}^{T} \boldsymbol{\sigma}_{e}\right)^{2}} S\left(\boldsymbol{\sigma}_{e}\right)+\frac{8}{\left(1+\boldsymbol{\sigma}_{e}^{T} \boldsymbol{\sigma}_{e}\right)^{2}} S^{2}\left(\boldsymbol{\sigma}_{e}\right) .
$$

Furthermore, based on the fact that $\dot{R}=-S\left(\boldsymbol{\omega}_{e}\right) R$, calculating the time derivative of relative pose and velocity results in the relative kinematics and relative dynamics expressed in frame $\mathcal{F}_{c}$ as

$$
\left\{\begin{array}{l}
\dot{\boldsymbol{p}}_{e}=\mathcal{A}_{e} \boldsymbol{p}_{e}+\mathcal{B}_{e} \boldsymbol{q}_{e} \\
\mathcal{M}_{e} \dot{\boldsymbol{q}}_{e}+\mathcal{C}_{e} \boldsymbol{q}_{e}+\boldsymbol{g}_{e}=\boldsymbol{u}+\boldsymbol{d}_{e}
\end{array}\right.
$$

where $\boldsymbol{p}_{e}=\left[\boldsymbol{r}_{e}, \boldsymbol{\sigma}_{e}^{T}\right]^{T}, \boldsymbol{q}_{e}=\left[\boldsymbol{v}_{e}^{T}, \boldsymbol{\omega}_{e}^{T}\right]^{T}, \mathcal{A}_{e}=\mathcal{A}, \mathcal{B}_{e}=$ $\operatorname{diag}\left\{I_{3}, G\left(\boldsymbol{\sigma}_{e}\right)\right\}, \mathcal{M}_{e}=\mathcal{M}, \mathcal{C}_{e}=\mathcal{C}, \boldsymbol{g}_{e}=\left(\mathcal{M S}_{e}+\mathcal{C}\right)(\boldsymbol{q}-$ $\left.\boldsymbol{q}_{e}\right), \boldsymbol{d}_{e}=\mathcal{E} \boldsymbol{u}+\left(I_{6}+\mathcal{E}\right) \boldsymbol{d}+\mathcal{M} \mathcal{R}_{e} \mathcal{M}_{t}^{-1}\left[\mathcal{C}_{t} \mathcal{R}_{e}^{T}\left(\boldsymbol{q}-\boldsymbol{q}_{e}\right)-\mathcal{E}_{t} \boldsymbol{d}_{t}\right]$, $\mathcal{S}_{e}=\operatorname{diag}\left\{S\left(\boldsymbol{\omega}_{e}\right), S\left(\boldsymbol{\omega}_{e}\right)\right\}$, and $\mathcal{R}_{e}=\operatorname{diag}\{R, R\}$.

Remark 1: The matrix $\mathcal{M}_{e}$ is constant, symmetric and positive definite, namely $\dot{\mathcal{M}}_{e}=O_{6}$ and $\mathcal{M}_{e}=\mathcal{M}_{e}^{T}>$ 0 . Meanwhile, the matrix $\mathcal{C}_{e}$ is skew-symmetric such that $\mathcal{C}_{e}=-\mathcal{C}_{e}^{T}$, since $\mathcal{C}=-\mathcal{C}^{T}$ and $S(\boldsymbol{\omega}) J-m S(\boldsymbol{\omega}) S^{2}(\boldsymbol{l})=$ $-S\left(\left(J-m S^{2}(\boldsymbol{l})\right) \boldsymbol{\omega}\right)$ in (1).

Remark 2: The applicability of the model (6) is explained slightly here. In Figure 1, the chaser and target can be viewed as two rigid spacecraft in the outer space or two rigid underwater vehicles in the sea, while the target are uncontrolled and disturbed by the environmental interferences. Then the non-cooperative close-range proximity and docking missions can be modeled by using the proposed relative pose dynamics (6), where two feature points can be viewed as the docking ports of two spacecraft or underwater vehicles. Thus, the relative translational and relative rotational motions between two docking ports can be described by the model (6). Furthermore, the linear model may not be straightforward in a wide class of real systems, in particular for the complicated docking missions in engineering. Thus the nonlinear model for the relative pose motion between two feature points is established in (6), and the dynamical couplings, input saturation and model uncertainties are also considered in the proposed dynamics and controller design.

Before the statement of the control objectives of this study, the following assumptions are claimed.

Assumption 1: $m$ and $J$ are uncertain but constant, while $m_{t}$ and $J_{t}$ are completely unknown but constant. Furthermore, the chaser's parameters can be treated as $m=m_{0}+m_{\Delta}$ and $J=J_{0}+J_{\Delta}$ with the known parts $m_{0}$ and $J_{0}$ and the unknown parts $m_{\Delta}$ and $J_{\Delta}$. The external disturbances are unknown but bounded by $\|\boldsymbol{d}\| \leq \bar{d}$ and $\left\|\boldsymbol{d}_{t}\right\| \leq \bar{d}_{t}$ with unknown constants $\bar{d}$ and $\bar{d}_{t}$, respectively. The constant vectors $\boldsymbol{l}$ and $\boldsymbol{l}_{t}$ are unknown by the chaser.

Assumption 2: The chaser is active for relative pose control with the passive target in this study, while the chaser can obtained precisely its motion information $\{\boldsymbol{p}, \boldsymbol{q}\}$ and relative motion information $\left\{\boldsymbol{p}_{e}, \boldsymbol{q}_{e}\right\}$ in real time based on the highperformance measurement devices and equipments which are mounted on the body of the chaser.

This paper aims to develop an advanced control command $\boldsymbol{u}_{0}$ under Assumptions 1 and 2 to ensure the precisely relative position tracking and attitude synchronization between feature points of two rigid bodies. This objective can be quantified accurately that the system states $\boldsymbol{p}_{e}(t)$ and $\boldsymbol{q}_{e}(t)$ are ultimately uniformly bounded convergent as $t \rightarrow \infty$. Meanwhile, the control input saturation is never violated, and the output constraints are never violated, namely $\left|p_{e k}(t)\right|<\bar{p}_{e k}$ for all $t \geq 0$ with prescribed constants $\bar{p}_{e k}$ if the initial outputs satisfy $\left|p_{e k}(0)\right|<\bar{p}_{e k}(k=1,2, \cdots, 6)$. 


\section{Controller Design And Stability AnAlysis}

\section{A. Controller Design}

Define the backstepping variables

$$
\boldsymbol{z}_{1}=\boldsymbol{p}_{e}, \quad \boldsymbol{z}_{2}=\boldsymbol{q}_{e}-\boldsymbol{\alpha}
$$

where $\alpha$ is the virtual stabilizing function to be designed. Then the relative pose dynamics (6) can be rewritten as

$$
\left\{\begin{array}{l}
\dot{\boldsymbol{z}}_{1}=\mathcal{A}_{e} \boldsymbol{z}_{1}+\mathcal{B}_{e}\left(\boldsymbol{z}_{2}+\boldsymbol{\alpha}\right) \\
\mathcal{M}_{e} \dot{\boldsymbol{z}}_{2}+\mathcal{C}_{e} \boldsymbol{z}_{2}+\boldsymbol{g}_{e}+\mathcal{M}_{e} \dot{\boldsymbol{\alpha}}=\boldsymbol{u}+\boldsymbol{d}_{e}
\end{array}\right.
$$

To overcome the output constraints of the relative kinematics and design the virtual stabilizing function, a candidate barrier Lyapunov function is chosen firstly as

$$
v_{1}=\sum_{k=1}^{6} \frac{1}{2} \log \frac{\overline{\boldsymbol{p}}_{e}^{T} E_{k} \overline{\boldsymbol{p}}_{e}}{\overline{\boldsymbol{p}}_{e}^{T} E_{k} \overline{\boldsymbol{p}}_{e}-\boldsymbol{z}_{1}^{T} E_{k} \boldsymbol{z}_{1}}
$$

where $\overline{\boldsymbol{p}}_{e}=\left[\bar{p}_{e 1}, \bar{p}_{e 2}, \cdots, \bar{p}_{e 6}\right]^{T}$ is the constraint vector of the system output $\boldsymbol{z}_{1} ; E_{k}$ denotes a $6 \times 6$ square matrix, where only the $k$ th diagonal entry is equal to one while all the other entries are zeros. Then the time derivative of $v_{1}$ is

$$
\dot{v}_{1}=\sum_{k=1}^{6} \frac{\boldsymbol{z}_{1}^{T} E_{k}\left[\mathcal{A}_{e} \boldsymbol{z}_{1}+\mathcal{B}_{e}\left(\boldsymbol{z}_{2}+\boldsymbol{\alpha}\right)\right]}{\overline{\boldsymbol{p}}_{e}^{T} E_{k} \overline{\boldsymbol{p}}_{e}-\boldsymbol{z}_{1}^{T} E_{k} \boldsymbol{z}_{1}}
$$

Since the matrix $\mathcal{B}_{e}$ is invertible because of non-singularity of Jaccobian matrix $G\left(\boldsymbol{\sigma}_{e}\right)$ in $\mathcal{B}_{e}$, then the virtual stabilizing function can be designed by

$$
\boldsymbol{\alpha}=-\mathcal{B}_{e}^{-1} K_{1} \boldsymbol{z}_{1}
$$

with the positive-definite diagonal gain matrix $K_{1}$. Meanwhile, its time derivative can be explicitly calculated by

$$
\dot{\boldsymbol{\alpha}}=-\dot{\mathcal{B}}_{e}^{-1} K_{1} \boldsymbol{z}_{1}-\mathcal{B}_{e}^{-1} K_{1}\left[\mathcal{A}_{e} \boldsymbol{z}_{1}+\mathcal{B}_{e}\left(\boldsymbol{z}_{2}+\boldsymbol{\alpha}\right)\right]
$$

where $\dot{\mathcal{B}}_{e}^{-1}=\operatorname{diag}\left\{O_{3}, \dot{G}^{-1}\left(\boldsymbol{\sigma}_{e}\right)\right\}$ and $\dot{G}^{-1}\left(\boldsymbol{\sigma}_{e}\right)=$ $-G^{-1}\left(\boldsymbol{\sigma}_{e}\right) \dot{G}\left(\boldsymbol{\sigma}_{e}\right) G^{-1}\left(\boldsymbol{\sigma}_{e}\right)$ with [35]

$$
\begin{aligned}
\dot{G}\left(\boldsymbol{\sigma}_{e}\right)= & \frac{8}{\left(1+\boldsymbol{\sigma}_{e}^{\mathrm{T}} \boldsymbol{\sigma}_{e}\right)^{2}}\left[\dot{\boldsymbol{\sigma}}_{e} \boldsymbol{\sigma}_{e}^{\mathrm{T}}+\boldsymbol{\sigma}_{e} \dot{\boldsymbol{\sigma}}_{e}^{\mathrm{T}}-\dot{\boldsymbol{\sigma}}_{e}^{\mathrm{T}} \boldsymbol{\sigma}_{e} I_{3}-S\left(\dot{\boldsymbol{\sigma}}_{e}\right)\right]- \\
& \frac{16 \boldsymbol{\sigma}_{e}^{\mathrm{T}} \dot{\boldsymbol{\sigma}}_{e}}{\left(1+\boldsymbol{\sigma}_{e}^{\mathrm{T}} \boldsymbol{\sigma}_{e}\right)^{3}}\left[\left(1-\boldsymbol{\sigma}_{e}^{\mathrm{T}} \boldsymbol{\sigma}_{e}\right) I_{3}-2 S\left(\boldsymbol{\sigma}_{e}\right)+2 \boldsymbol{\sigma}_{e} \boldsymbol{\sigma}_{e}^{\mathrm{T}}\right] .
\end{aligned}
$$

Then, due to the skew-symmetric property of the matrix $\mathcal{A}_{e}$, substituting (10) into (28) results in

$$
\dot{v}_{1}=\sum_{k=1}^{6} \frac{-\boldsymbol{z}_{1}^{T} E_{k} K_{1} \boldsymbol{z}_{1}+\boldsymbol{z}_{1}^{T} E_{k} \mathcal{B}_{e} \boldsymbol{z}_{2}}{\overline{\boldsymbol{p}}_{e}^{T} E_{k} \overline{\boldsymbol{p}}_{e}-\boldsymbol{z}_{1}^{T} E_{k} \boldsymbol{z}_{1}}
$$

In view of the parametric uncertainties in Assumption 1, the second sub-equation of (7) can be rewritten as

$$
\mathcal{M}_{e} \dot{\boldsymbol{z}}_{2}=-\mathcal{M}_{e 0} \dot{\boldsymbol{\alpha}}-\mathcal{C}_{e 0} \boldsymbol{z}_{2}-\boldsymbol{g}_{e 0}+\boldsymbol{u}+\boldsymbol{w}
$$

where $\boldsymbol{w}=\boldsymbol{d}_{e}-\mathcal{M}_{e \Delta} \dot{\boldsymbol{\alpha}}-\mathcal{C}_{e \Delta} \boldsymbol{z}_{2}-\boldsymbol{g}_{e \Delta}$;

$$
\begin{gathered}
\mathcal{M}_{e j}=\left[\begin{array}{cc}
m_{j} I_{3} & -m_{j} S(\boldsymbol{l}) \\
m_{j} S(\boldsymbol{l}) & J_{j}-m_{j} S^{2}(\boldsymbol{l})
\end{array}\right] ; \\
\mathcal{C}_{e j}=\left[\begin{array}{cc}
m_{j} S(\boldsymbol{\omega}) & -m_{j} S(\boldsymbol{\omega}) S(\boldsymbol{l}) \\
m_{j} S(\boldsymbol{l}) S(\boldsymbol{\omega}) & S(\boldsymbol{\omega}) J_{j}-m_{j} S(\boldsymbol{\omega}) S^{2}(\boldsymbol{l})
\end{array}\right] ;
\end{gathered}
$$

$\boldsymbol{g}_{e j}=\left(\mathcal{M}_{e j} \mathcal{S}_{e}+\mathcal{C}_{e j}\right)\left(\boldsymbol{q}-\boldsymbol{q}_{e}\right) ;(j=0, \Delta)$. Furthermore, due to the unknown misalignment vector $\boldsymbol{l}$ in $\mathcal{M}_{e 0}, \mathcal{C}_{e 0}$ and $\boldsymbol{g}_{e 0}$, the equation (13) can be further rewritten as

$$
\mathcal{M}_{e} \dot{z}_{2}=\boldsymbol{h}_{a}+\boldsymbol{h}_{b}+\boldsymbol{u}+\boldsymbol{w}
$$

where $\boldsymbol{h}_{a}=-\mathcal{M}_{e 0}^{a}\left[\dot{\boldsymbol{\alpha}}+\mathcal{S}_{e}\left(\boldsymbol{q}-\boldsymbol{q}_{e}\right)\right]-\mathcal{C}_{e 0}^{a}\left(\boldsymbol{z}_{2}+\boldsymbol{q}-\boldsymbol{q}_{e}\right)$, $\boldsymbol{h}_{b}=-\mathcal{M}_{e 0}^{b}\left[\dot{\boldsymbol{\alpha}}+\mathcal{S}_{e}\left(\boldsymbol{q}-\boldsymbol{q}_{e}\right)\right]-\mathcal{C}_{e 0}^{b}\left(\boldsymbol{z}_{2}+\boldsymbol{q}-\boldsymbol{q}_{e}\right)$; and

$$
\begin{gathered}
\mathcal{M}_{e 0}^{a}=\left[\begin{array}{cc}
m_{0} I_{3} & O_{3} \\
O_{3} & J_{0}
\end{array}\right] ; \mathcal{C}_{e 0}^{a}=\left[\begin{array}{cc}
m_{0} S(\boldsymbol{\omega}) & O_{3} \\
O_{3} & S(\boldsymbol{\omega}) J_{0}
\end{array}\right] ; \\
\mathcal{M}_{e 0}^{b}=\left[\begin{array}{cc}
O_{3} & -m_{0} S(\boldsymbol{l}) \\
m_{0} S(\boldsymbol{l}) & -m_{0} S^{2}(\boldsymbol{l})
\end{array}\right] ; \\
\mathcal{C}_{e 0}^{b}=\left[\begin{array}{cc}
O_{3} & -m_{0} S(\boldsymbol{\omega}) S(\boldsymbol{l}) \\
m_{0} S(\boldsymbol{l}) S(\boldsymbol{\omega}) & -m_{0} S(\boldsymbol{\omega}) S^{2}(\boldsymbol{l})
\end{array}\right] .
\end{gathered}
$$

To deal with the unknown vector $\boldsymbol{l}=\left[l_{1}, l_{2}, l_{3}\right]^{T}$, a linear operator is introduced for any $\boldsymbol{x}=\left[x_{1}, x_{2}, x_{3}\right]^{T} \in \mathbb{R}^{3}$ as

$$
L(\boldsymbol{x})=\left[\begin{array}{cccccc}
x_{1} & x_{2} & x_{3} & 0 & 0 & 0 \\
0 & x_{1} & 0 & x_{2} & x_{3} & 0 \\
0 & 0 & x_{1} & 0 & x_{2} & x_{3}
\end{array}\right]
$$

such that $S^{2}(\boldsymbol{l}) \boldsymbol{x}=L(\boldsymbol{x}) \boldsymbol{l}_{\theta}$ with $\boldsymbol{l}_{\theta}=\left[-\left(l_{2}^{2}+\right.\right.$ $\left.\left.l_{3}^{2}\right), l_{1} l_{2}, l_{1} l_{3},-\left(l_{1}^{2}+l_{3}^{2}\right), l_{2} l_{3},-\left(l_{1}^{2}+l_{2}^{2}\right)\right]^{T}$. Then the actuator saturation and the fact $S(\boldsymbol{l}) \boldsymbol{x}=-S(\boldsymbol{x}) \boldsymbol{l}$ lead to

$$
\mathcal{M}_{e} \dot{z}_{2}=\boldsymbol{h}_{a}+Y \boldsymbol{\theta}+\boldsymbol{u}_{0}+\boldsymbol{u}_{\Delta}+\boldsymbol{w}
$$

where $\boldsymbol{\theta}=\left[\boldsymbol{l}^{T}, \boldsymbol{l}_{\theta}^{T}\right]^{T} \in \mathbb{R}^{9} ; \boldsymbol{u}_{\Delta}=\boldsymbol{u}-\boldsymbol{u}_{0} ; Y=$ $\left[Y_{11}, O_{3 \times 6} ; Y_{21}, Y_{22}\right] \in \mathbb{R}^{6 \times 9} ;$ and $Y_{11}=-m_{0} S\left(\boldsymbol{\beta}_{2}\right)-$ $m_{0} S(\boldsymbol{\omega}) S\left(\boldsymbol{\gamma}_{2}\right), Y_{21}=m_{0} S\left(\boldsymbol{\beta}_{1}\right)+m_{0} S\left(S(\boldsymbol{\omega}) \boldsymbol{\gamma}_{1}\right), Y_{22}=$ $m_{0} L\left(\boldsymbol{\beta}_{2}\right)+m_{0} S(\boldsymbol{\omega}) L\left(\boldsymbol{\gamma}_{2}\right) ; \boldsymbol{\beta}_{1} \in \mathbb{R}^{3}$ and $\boldsymbol{\beta}_{2} \in \mathbb{R}^{3}$ are the first and the last three entries of vector $\boldsymbol{\beta} \triangleq \dot{\boldsymbol{\alpha}}+\mathcal{S}_{e}\left(\boldsymbol{q}-\boldsymbol{q}_{e}\right)$, respectively; $\gamma_{1} \in \mathbb{R}^{3}$ and $\gamma_{2} \in \mathbb{R}^{3}$ are the first and the last three entries of vector $\gamma \triangleq \boldsymbol{z}_{2}+\boldsymbol{q}-\boldsymbol{q}_{e}=\boldsymbol{q}-\boldsymbol{\alpha}$, respectively; $\boldsymbol{f}_{0}$ and $\boldsymbol{f}_{\Delta}$ are the first three entries of vectors $\boldsymbol{u}_{0}$ and $\boldsymbol{u}_{\Delta}$, respectively.

To deal with the actuator saturation effect $\boldsymbol{u}_{\Delta}$ in (15), the linear anti-windup compensator is designed as

$$
\dot{\zeta}=-\Pi \zeta+u_{\Delta}
$$

where the positive-definite gain matrix $\Pi$. Meanwhile, in order to deal with the lumped disturbance $\boldsymbol{w}$ including unknown dynamical couplings and external disturbances of two rigid bodies in (15), it is assumed that $\|\boldsymbol{w}\| \leq \bar{w}$ with an unknown constant $\bar{w}$, and the direct adaptive hierarchical fuzzy logic system is employed to approximate the unknown term $\boldsymbol{w}$ such that

$$
\boldsymbol{w}=\boldsymbol{w}^{*}(s)+\boldsymbol{\epsilon}, \quad\|\boldsymbol{\epsilon}\| \leq \bar{\epsilon}
$$

where $\boldsymbol{s}=\left[\boldsymbol{z}_{1}^{T}, \boldsymbol{z}_{2}^{T}, \boldsymbol{q}\right]^{T}=\left[s_{1}, s_{2}, \cdots, s_{18}\right]^{T} \in \mathbb{R}^{18}$ is the input vector of the adaptive fuzzy logic system; $\boldsymbol{w}^{*}(\boldsymbol{s})$ is the optimal approximation of $\boldsymbol{w} ; \boldsymbol{\epsilon}$ is the minimum approximation error; $\bar{\epsilon}$ is an unknown constant. In addition, define the secondorder smooth nonlinear function

$$
\rho(\|\boldsymbol{s}\|)= \begin{cases}0 & \|\boldsymbol{s}\| \leq b_{1} \\ 1-\cos ^{2}\left[\frac{\pi}{2} \sin ^{2}\left(\frac{\pi}{2} \frac{\|\boldsymbol{s}\|^{2}-b_{1}^{2}}{b_{2}^{2}-b_{1}^{2}}\right)\right] & b_{1}<\|\boldsymbol{s}\|<b_{2} \\ 1 & \|s\| \geq b_{2}\end{cases}
$$


where $0<b_{1}<b_{2} ; b_{1}$ and $b_{2}$ are tuning parameters given by the designer. Then, the controller can be designed as

$$
\begin{aligned}
\boldsymbol{u}_{0}= & -\sum_{i=1}^{6} \frac{\mathcal{B}_{e}^{T} E_{i}^{T} \boldsymbol{z}_{1}}{\overline{\boldsymbol{p}}_{e}^{T} E_{i} \overline{\boldsymbol{p}}_{e}-\boldsymbol{z}_{1}^{T} E_{i} \boldsymbol{z}_{1}}-K_{2} \boldsymbol{z}_{2}-K_{3} \boldsymbol{\zeta} \\
& -\boldsymbol{h}_{a}-Y \hat{\boldsymbol{\theta}}+[1-\rho(\|\boldsymbol{s}\|)] \boldsymbol{\eta}_{n}+\rho(\|\boldsymbol{s}\|) \boldsymbol{\eta}_{t}
\end{aligned}
$$

with

$$
\boldsymbol{\eta}_{n}=-\hat{\boldsymbol{w}}-\hat{\bar{\epsilon}} \tanh \left(\nu \boldsymbol{z}_{2}\right), \quad \boldsymbol{\eta}_{t}=-\hat{\bar{w}} \tanh \left(\nu \boldsymbol{z}_{2}\right)
$$

where $K_{2}$ and $K_{3}$ are positive-definite gain matrices; $\hat{\boldsymbol{\theta}}$ is the estimation of $\boldsymbol{\theta} ; \hat{\boldsymbol{w}}$ is the fuzzy control; $\tanh (\nu \boldsymbol{x}) \triangleq$ $\left[\tanh \left(\nu x_{1}\right), \tanh \left(\nu x_{2}\right), \cdots, \tanh \left(\nu x_{n}\right)\right]^{T}$ for any vector $\boldsymbol{x}=$ $\left[x_{1}, x_{2}, \cdots, x_{n}\right]^{T}$, and $\nu>0$ is a positive gain constant; the parameters $b_{1}$ and $b_{2}$ in function $\rho(\|\boldsymbol{s}\|)$ determine the transient progress between the region of the adaptive fuzzy logic systems and the region of the robust adaptive compensation control. In particular, the unknown parameters $\boldsymbol{\theta}, \bar{\epsilon}$ and $\bar{w}$ are estimated by the following adaptive laws

$$
\left\{\begin{array}{l}
\dot{\hat{\boldsymbol{\theta}}}=-c_{1} c_{2} \hat{\boldsymbol{\theta}}+c_{2} Y^{T} \boldsymbol{z}_{2} \\
\dot{\hat{\bar{\epsilon}}}=-c_{3} c_{4} \hat{\bar{\epsilon}}+c_{4}(1-\rho) \boldsymbol{z}_{2}^{T} \tanh \left(\nu \boldsymbol{z}_{2}\right) \\
\dot{\hat{\bar{w}}}=-c_{5} c_{6} \hat{\bar{w}}+c_{6} \rho \boldsymbol{z}_{2}^{T} \tanh \left(\nu \boldsymbol{z}_{2}\right)
\end{array}\right.
$$

with positive gain constants $c_{k}>0(k=1,2, \cdots, 6)$. The fuzzy control $\hat{\boldsymbol{w}}$ is derived by using six adaptive hierarchical fuzzy logic systems as

$$
\hat{\boldsymbol{w}}(\boldsymbol{s}, \hat{\boldsymbol{\vartheta}})=\left[\begin{array}{c}
\hat{w}_{1}\left(\boldsymbol{s}, \hat{\boldsymbol{\vartheta}}_{1}\right) \\
\hat{w}_{2}\left(\boldsymbol{s}, \hat{\boldsymbol{\vartheta}}_{2}\right) \\
\hat{w}_{3}\left(\boldsymbol{s}, \hat{\boldsymbol{\vartheta}}_{3}\right) \\
\hat{w}_{4}\left(\boldsymbol{s}, \hat{\boldsymbol{\vartheta}}_{4}\right) \\
\hat{w}_{5}\left(\boldsymbol{s}, \hat{\boldsymbol{\vartheta}}_{5}\right) \\
\hat{w}_{6}\left(\boldsymbol{s}, \hat{\boldsymbol{\vartheta}}_{6}\right)
\end{array}\right]=\left[\begin{array}{c}
\hat{\boldsymbol{\vartheta}}_{1,17}^{T} \boldsymbol{\phi}_{1,17}\left(y_{1,16}, s_{18}\right) \\
\hat{\boldsymbol{\vartheta}}_{2,17}^{T} \boldsymbol{\phi}_{2,17}\left(y_{2,16}, s_{18}\right) \\
\hat{\boldsymbol{\vartheta}}_{3,17}^{T} \boldsymbol{\phi}_{3,17}\left(y_{3,16}, s_{18}\right) \\
\hat{\boldsymbol{\vartheta}}_{4,17}^{T} \boldsymbol{\phi}_{4,17}\left(y_{4,16}, s_{18}\right) \\
\hat{\boldsymbol{\vartheta}}_{5,17}^{T} \boldsymbol{\phi}_{5,17}\left(y_{5,16}, s_{18}\right) \\
\hat{\boldsymbol{\vartheta}}_{6,17}^{T} \boldsymbol{\phi}_{6,17}\left(y_{6,16}, s_{18}\right)
\end{array}\right]
$$

where $\hat{\boldsymbol{\vartheta}}_{k}=\left[\hat{\boldsymbol{\vartheta}}_{k, 1}^{T}, \hat{\boldsymbol{\vartheta}}_{k, 2}^{T}, \cdots, \hat{\boldsymbol{\vartheta}}_{k, 17}^{T}\right]^{T}, \quad y_{k, 16}=$ $\hat{\boldsymbol{\vartheta}}_{k, 16}^{T} \boldsymbol{\phi}_{k, 16}\left(y_{k, 15}, s_{17}\right), \quad y_{k, 15}=\hat{\boldsymbol{\vartheta}}_{k, 15}^{T} \boldsymbol{\phi}_{k, 15}\left(y_{k, 14}, s_{16}\right)$, $y_{k, 14}=\hat{\boldsymbol{\vartheta}}_{k, 14}^{T} \phi_{k, 14}\left(y_{k, 13}, s_{15}\right), \cdots, y_{k, 1}=\hat{\boldsymbol{\vartheta}}_{k, 1}^{T} \phi_{k, 1}\left(s_{1}, s_{2}\right)$. Here the structure of the $k(k=1,2, \cdots, 6)$ th adaptive hierarchical fuzzy logic systems is shown in Fig. 2, where the first two state variables are used as the inputs of the first-level fuzzy subsystem, then its output and the third state variable are used as the inputs of the second-level fuzzy subsystem, and this process is repeated in succeeding levels of hierarchy until all state variables in the vector $s$ in (17) are used completely. The first-level fuzzy subsystem is established by the IF-THEN rule:

$$
\text { IF } \hat{s}_{1} \text { is } A_{1}^{j} \text {, and } \hat{s}_{2} \text { is } A_{2}^{j} \text {, THEN } y_{k, 1} \text { is } B_{1}^{j}
$$

where $\hat{s}_{i}(i=1,2)$ is the fuzzy variables by fuzzing input variables $s_{i}$; every fuzzy variable $s_{i}$ has five fuzzy sets such that the total amount of the fuzzy rules in the first-level fuzzy subsystem is $25 ; A_{i}^{j}(i=1,2)$ and $B_{1}^{j}$ are fuzzy sets, and the corresponding $\mu_{A_{i}^{j}}\left(s_{i}\right)$ and $\mu_{B^{l}}\left(y_{k, 1}\right)$ are Gaussian-type membership functions. If the fuzzy subsystems are conducted

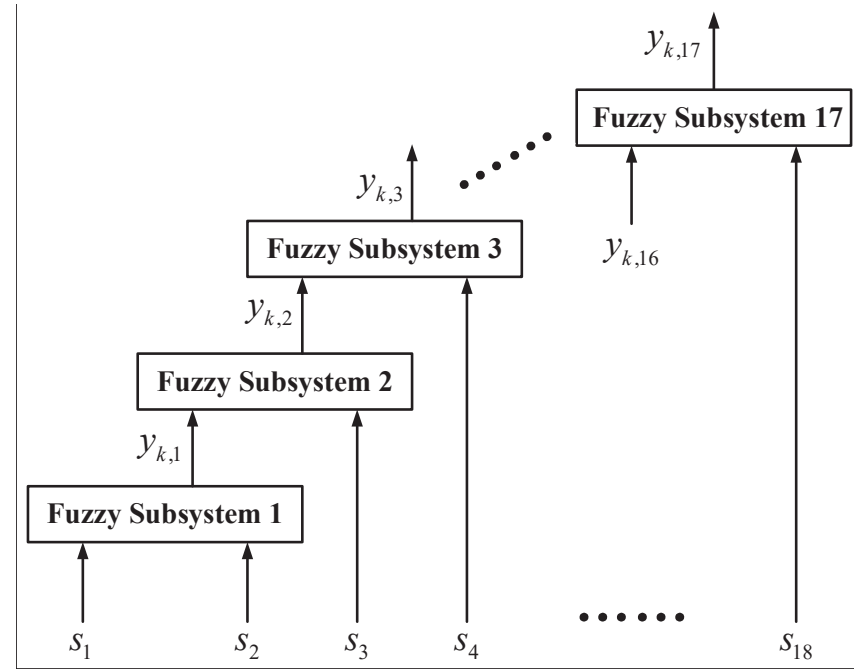

Fig. 2. The structure of the $k$ th adaptive hierarchical fuzzy logic system.

with singleton fuzzifier, center-average defuzzifier, and product inference engine, then the output of the first-level fuzzy subsystem $y_{k, 1}$ can be expressed by

$$
y_{k, 1}=\frac{\sum_{j=1}^{25} \hat{\vartheta}_{1, j} \mu_{A_{1}^{j}}\left(s_{1}\right) \mu_{A_{2}^{j}}\left(s_{2}\right)}{\sum_{j=1}^{25} \mu_{A_{1}^{j}}\left(s_{1}\right) \mu_{A_{2}^{j}}\left(s_{2}\right)}=\hat{\boldsymbol{\vartheta}}_{k, 1}^{T} \phi_{k, 1}\left(s_{1}, s_{2}\right)
$$

where $\hat{\vartheta}_{k, 1}=\left[\hat{\vartheta}_{1,1}, \cdots, \hat{\vartheta}_{1,25}\right]^{T}, \quad \hat{\vartheta}_{1, j}$ is the estimation of the point at which the membership function of $\mu_{B_{1}^{j}}\left(y_{k, 1}\right)$ achieves its maximum value; $\phi_{k, 1}\left(s_{1}, s_{2}\right)=$ $\left[\phi_{1,1}\left(s_{1}, s_{2}\right), \cdots, \phi_{1,25}\left(s_{1}, s_{2}\right)\right]^{T}$ with the fuzzy basis function

$$
\phi_{1, j}\left(s_{1}, s_{2}\right)=\frac{\mu_{A_{1}^{j}}\left(s_{1}\right) \mu_{A_{2}^{j}}\left(s_{2}\right)}{\sum_{j=1}^{25} \mu_{A_{1}^{j}}\left(s_{1}\right) \mu_{A_{2}^{j}}\left(s_{2}\right)}
$$

Furthermore, the $i(i=2, \cdots, 17)$ th-level fuzzy subsystem is established by the following IF-THEN rules:

$$
\text { IF } \hat{s}_{i+1} \text { is } A_{i+1}^{j} \text {, and } \hat{y}_{k, i-1} \text { is } B_{i-1}^{j} \text {, THEN } y_{k, i} \text { is } B_{i}^{j}
$$

where $\hat{s}_{i+1}$ and $\hat{y}_{k, i-1}$ are the fuzzifier values of inputs $s_{i+1}$ and $y_{k, i-1}$, respectively. $A_{i+1}^{j}, B_{i-1}^{j}$, and $B_{i}^{j}$ are fuzzy sets, and their membership functions $\mu_{A_{i+1}^{j}}\left(s_{i+1}\right), \mu_{B_{i-1}^{j}}\left(y_{k, i-1}\right)$, and $\mu_{B_{i}^{j}}\left(y_{k, i}\right)$ are also Gaussian types. Following the first-level fuzzy subsystem, the $i(i=2, \cdots, 17)$ th-level fuzzy subsystem output $y_{k, i}$ is

$$
\begin{aligned}
y_{k, i} & =\frac{\sum_{j=1}^{25} \hat{\vartheta}_{i, j} \mu_{B_{i-1}^{j}}\left(y_{k, i-1}\right) \mu_{A_{i+1}^{j}}\left(s_{i+1}\right)}{\sum_{j=1}^{25} \mu_{B_{i-1}^{j}}\left(y_{k, i-1}\right) \mu_{A_{i+1}^{j}}\left(s_{i+1}\right)} \\
& =\hat{\boldsymbol{\vartheta}}_{k, i}^{T} \phi_{k, i}\left(y_{k, i-1}, s_{i+1}\right)
\end{aligned}
$$

Let $y_{0}=s_{1}$ and $\mu_{B_{0}}^{j}=\mu_{A_{1}}^{j}$, then the total output of the $k$ th adaptive hierarchical fuzzy system is

$$
y_{k, 17}=\hat{\boldsymbol{\vartheta}}_{k, 17}^{T} \phi_{k, 17}\left(y_{k, 16}, s_{18}\right)=\hat{w}_{k}\left(\boldsymbol{s}, \hat{\boldsymbol{\vartheta}}_{k}\right)
$$

where $y_{k, 16}$ is determined by (22). 
The projection-based parameter adaptive law for the fuzzy logic systems is designed as

$\dot{\hat{\boldsymbol{\vartheta}}}_{k, j}= \begin{cases}\kappa_{\rho} z_{2 k} \boldsymbol{\phi}_{k, j}, & \left\|\hat{\boldsymbol{\vartheta}}_{k, j}\right\|<\Theta_{k, j} ; \\ & \left\|\hat{\boldsymbol{\vartheta}}_{k, j}\right\|=\Theta_{k, j}, z_{2 k} \hat{\boldsymbol{\vartheta}}_{k, j}^{T} \boldsymbol{\phi}_{k, j} \leq 0 \\ \kappa_{\rho} z_{2 k}\left(\boldsymbol{\phi}_{k, j}-\boldsymbol{\kappa}_{\vartheta}\right), & \left\|\hat{\boldsymbol{\vartheta}}_{k, j}\right\|=\Theta_{k, j}, z_{2 k} \hat{\boldsymbol{\vartheta}}_{k, j}^{T} \boldsymbol{\phi}_{k, j}>0\end{cases}$

where $\boldsymbol{\kappa}_{\vartheta}=\frac{\hat{\boldsymbol{\vartheta}}_{k, j} \hat{\boldsymbol{\vartheta}}_{k, j}^{T} \boldsymbol{\phi}_{k, j}}{\left\|\hat{\boldsymbol{\vartheta}}_{k, j}\right\|^{2}} ; \kappa_{\rho}=\kappa(1-\rho) ; k=1, \cdots, 6 ; j=$ $1, \cdots, 17 ; \Theta_{k, j}$ and $\kappa$ are positive constants specified by the designer.

\section{B. Stability Analysis}

Theorem 1: Consider the relative motion model under the Assumptions 1 and 2. If a robust adaptive fuzzy controller (19) with anti-windup compensator (16) and adaptive laws (20) and (24) under the tunable rule $3 \bar{\lambda}_{K_{3}}^{2}<2 \underline{\lambda}_{K_{2}} \underline{\lambda}_{\Pi}$ and a mild assumption $\left\|\boldsymbol{u}_{\Delta}\right\| \leq \bar{u}_{\Delta}$ with unknown constant $\bar{u}_{\Delta}$ is used, then all signals in the closed-loop systems are always bounded. In addition, if the initial constraints $\left|p_{e k}(0)\right|<\bar{p}_{e k}(k=$ $1,2, \cdots, 6)$ are satisfied, then the constraints of the system outputs $p_{e k}(t)$ are never violated. Finally, the system states $\boldsymbol{p}_{e}$ and $\boldsymbol{q}_{e}$ are ultimately uniformly bounded.

Proof: The details of proof are given in the Appendix.

Remark 3: Compared with the previous study, the proposed control strategy has the following advantages. Firstly, if the constraints of the relative attitude are set as $\left|\sigma_{e i}\right|<1(i=$ $1,2,3)$, then the shadow-set description of the relative attitude parameters can be avoided and the non-singular relative attitude control can be guaranteed. Secondly, the combination of the adaptive hierarchical fuzzy system and the smooth function $\rho(\|s\|)$ in (18) can improve the robustness of the control system, since the state compact set for the proposed adaptive fuzzy system can be defined by the parameters $b_{1}$ and $b_{2}$ in $\rho(\|\boldsymbol{s}\|)$. Because of the approximation ability of the fuzzy logic system in the prescribed compact set, the unknown function in the model should be compensated by two mechanisms, the adaptive fuzzy logic system and robust adaptive control, where the adaptive fuzzy logic control is activated if the system state enters the prescribed compact set, and the robust adaptive control is activated if the system state leaves the prescribed compact set. Thus the transient progress between two mechanisms is defined by the secondorder smooth function. Thirdly, the hierarchical structure of the adaptive fuzzy logic system can greatly decrease the number of fuzzy rules for the six-degrees-of-freedom nonlinear system, since the total fuzzy rules of the proposed hierarchical fuzzy system is only $5^{2} \times 17 \times 6$ under five fuzzy sets for every input while the total fuzzy rules equals to $5^{18} \times 6$ for nonhierarchical structure. Lastly, the supervisor control strategy in the traditional adaptive fuzzy logic control methods can be avoided such that the upper-bound information of the model uncertainties can be avoided in the proposed method.

Remark 4: The stability of the closed-loop system is guaranteed by the proposed robust adaptive controller (19), where two kinds of adaptive mechanisms are used for estimating the unknown parameters in the system, such as the $\sigma$-modification adaptive method in (20) and the projectionbased adaptive method in (24). The adaptive estimations in (20) converge to the small neighborhoods of zero by tuning the parameters $c_{k}(k=1,2, \cdots, 6)$, and the adaptive estimations in (24) are always bounded in the prescribed compact sets $\Omega_{\vartheta}$ by the designer. Furthermore, the high steady-state precision of the system state $z_{s}$ is mainly determined by large $a_{s}$ and small $\varpi_{s}$. Thus, from the definition of $a_{s}$ and $\varpi_{s}$, one can conclude that the smaller neighborhoods of zero for the system states are resulted from the larger $K_{1}, K_{2}, \Pi, c_{2}, c_{4}, c_{6}, \nu, \kappa$ and smaller $c_{1}, c_{3}$, and $c_{5}$ under prescribed $\Theta_{k, j}$ and the tunable rule $3 \bar{\lambda}_{K_{3}}^{2}<2 \underline{\lambda}_{K_{2}} \underline{\lambda}_{\Pi}$.

\section{Simulation EXAMPLE}

Consider the relative pose motion between feature points of two rigid bodies in three-dimensional space, the true values of physical parameters about two rigid bodies are given by

$$
\begin{gathered}
m_{t}=5425.6(\mathrm{~kg}), \quad m=58.2(\mathrm{~kg}), \\
J_{t}=\left[\begin{array}{rrr}
3336.3 & -135.4 & -154.2 \\
-135.4 & 3184.5 & -148.5 \\
-154.2 & -148.5 & 2423.7
\end{array}\right]\left(\mathrm{kgm}^{2}\right), \\
J=\left[\begin{array}{rrr}
38.3 & -2.5 & -5.5 \\
-2.5 & 44.4 & -2.7 \\
-5.5 & -2.7 & 36.6
\end{array}\right]\left(\mathrm{kgm}^{2}\right), \\
m_{0}=60(\mathrm{~kg}), \quad J_{0}=\operatorname{diag}\{40,40,40\}\left(\mathrm{kgm}^{2}\right), \\
\boldsymbol{l}_{t}=[0,0.3,0]^{T}(\mathrm{~m}), \quad \boldsymbol{l}=[0.2,0,0]^{T}(\mathrm{~m}), \\
\boldsymbol{w}=0.03\left[\sin \left(\frac{\pi}{40} t\right), \cos \left(\frac{\pi}{40} t\right), \sin \left(\frac{\pi}{45} t\right)\right]^{T}(\mathrm{~N}), \\
\boldsymbol{\delta}=0.01\left[\sin \left(\frac{\pi}{45} t\right), \cos \left(\frac{\pi}{45} t\right), \sin \left(\frac{\pi}{40} t\right)\right]^{T}(\mathrm{Nm}), \\
\boldsymbol{w}_{t}=100 \boldsymbol{w}(\mathrm{N}), \quad \boldsymbol{\delta}_{t}=100 \boldsymbol{\delta}(\mathrm{Nm}) .
\end{gathered}
$$

The input and output constraints are prescribed by $\overline{\boldsymbol{p}}_{e}=$ $[5,5,5,1,1,1]^{T}$ and $\boldsymbol{u}_{\max }=\boldsymbol{u}_{\min }=[20,20,20,5,5,5]^{T}$. The initial conditions for system states and estimations are also given by $\boldsymbol{r}(0)=[10,12,10]^{T}(\mathrm{~m}), \boldsymbol{v}(0)=[2,3,-2]^{T}(\mathrm{~m} / \mathrm{s})$, $\boldsymbol{\sigma}(0)=\mathbf{0}, \boldsymbol{\omega}(0)=\mathbf{0}(\mathrm{rad} / \mathrm{s}), \boldsymbol{r}_{e}(0)=[-4.5,4.5,4.5]^{T}(\mathrm{~m})$, $\boldsymbol{v}_{e}(0)=[-0.2,-0.2,0.2]^{T}(\mathrm{~m} / \mathrm{s}), \boldsymbol{\sigma}_{e}(0)=[0.6,-0.7,0.7]^{T}$, $\boldsymbol{\omega}_{e}(0)=[0.02,0.02,0.02]^{T}, \hat{\boldsymbol{\theta}}(0)=\mathbf{0}, \hat{\bar{\epsilon}}(0)=\hat{\bar{w}}(0)=0$, $\hat{\boldsymbol{\vartheta}}_{k, j}(0)=\mathbf{0}$. The input vector $\boldsymbol{s}=\left[\boldsymbol{z}_{1}^{T}, \boldsymbol{z}_{2}^{T}, \boldsymbol{q}^{T}\right]^{T}$ of the hierarchical fuzzy systems shown in Figure 2 is mapped by $\hat{s}_{i}=\frac{s_{i}}{\left|s_{i}\right|+0.0001}(i=1,2, \cdots, 18)$ such that $-1<\hat{s}_{i}<1$. Five fuzzy sets are defined over the interval $\hat{s}_{i} \in(-1,1)$, and the corresponding membership functions are defined by

$$
\begin{gathered}
\mu_{A_{i}}^{1}=\mathrm{e}^{-\frac{1}{2}\left(\frac{\hat{s}_{i}-1}{0.25}\right)^{2}}, \mu_{A_{i}}^{2}=\mathrm{e}^{-\frac{1}{2}\left(\frac{\hat{s}_{i}-0.5}{0.25}\right)^{2}}, \mu_{A_{i}}^{3}=\mathrm{e}^{-\frac{1}{2}\left(\frac{\hat{s}_{i}}{0.25}\right)^{2}}, \\
\mu_{A_{i}}^{4}=\mathrm{e}^{-\frac{1}{2}\left(\frac{\hat{s}_{i}+0.5}{0.25}\right)^{2}}, \quad \mu_{A_{i}}^{5}=\mathrm{e}^{-\frac{1}{2}\left(\frac{\hat{s}_{i}+1}{0.25}\right)^{2}} .
\end{gathered}
$$

The tunable parameters are chosen as $K_{1}=0.1 I_{6}, K_{2}=$ $\operatorname{diag}\left\{450 I_{3}, 300 I_{3}\right\}, K_{3}=2 I_{6}, \Pi=5 I_{6}, c_{k}=0.2(k=$ $1,2, \cdots, 6), b_{1}=5, b_{2}=10, \kappa=0.05, \Theta_{k, j}=5, \nu=5$. 


\section{A. Effectiveness Validations}

The simulation results of the proposed control system are presented in Figures 3-6 based on the controller (19). Figure 3 shows the relative three-dimensional position and velocity between the feature points of two rigid bodies, and Figure 4 gives the results of attitude synchronization and relative angular velocity for these two rigid bodies. Figures 3 and 4 imply that the proposed control approach can achieve the designing objectives such that the relative pose coordination between feature points of two rigid bodies are with acceptable response performance. Figure 5 reveals a fact that the control force and torque inputs are always constrained in the prescribed limitations, and Figure 6 implies that the adaptive parameters and states of the anti-windup compensator are always bounded. In conclusion, the proposed controller is effective to complete the control objectives.
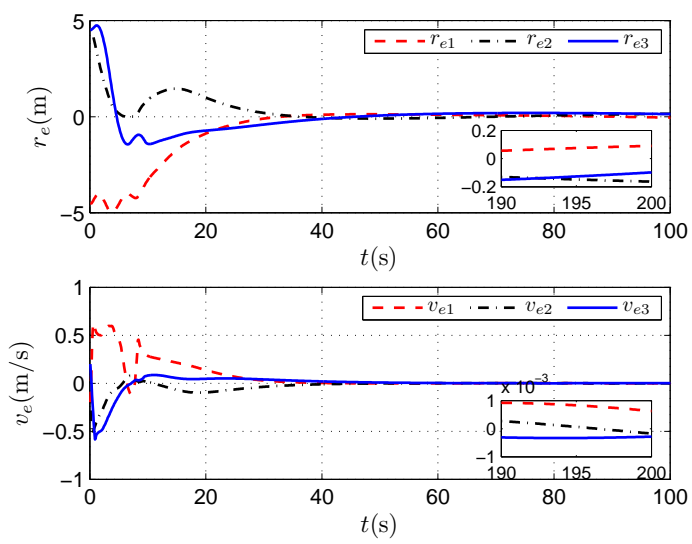

Fig. 3. Relative position and velocity by controller (19).
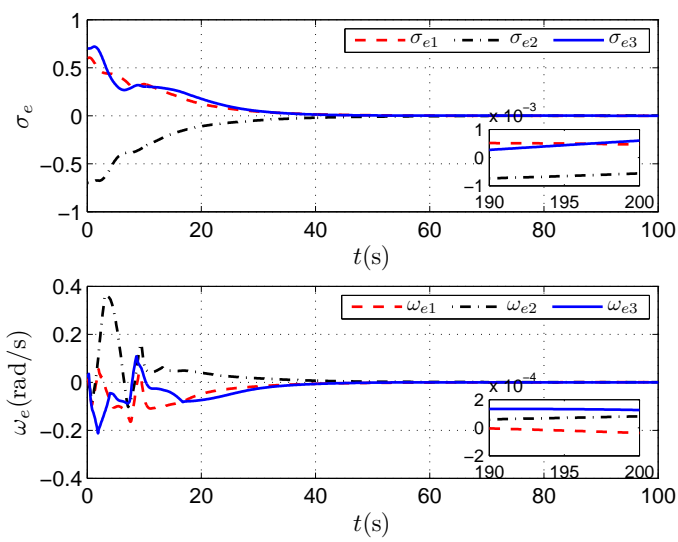

Fig. 4. Relative attitude and angular velocity by controller (19).

\section{B. Robustness Tests}

The robust performance of the proposed controller (19) should be tested under the large parametric uncertainties and external disturbances, where the nominal mass and inertia of matrix are reconsidered as $m_{0}=0.8 \mathrm{~m}$ and $J_{0}=0.8 \mathrm{~J}$, while the disturbances are set as $\boldsymbol{w}=\boldsymbol{w}_{0}+0.01 \operatorname{rand}(3,1)(\mathrm{N})$,
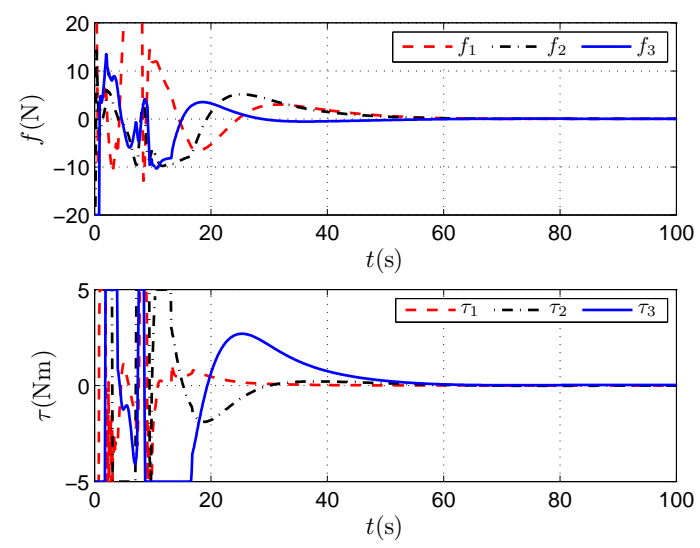

Fig. 5. Control forces and torques by controller (19).
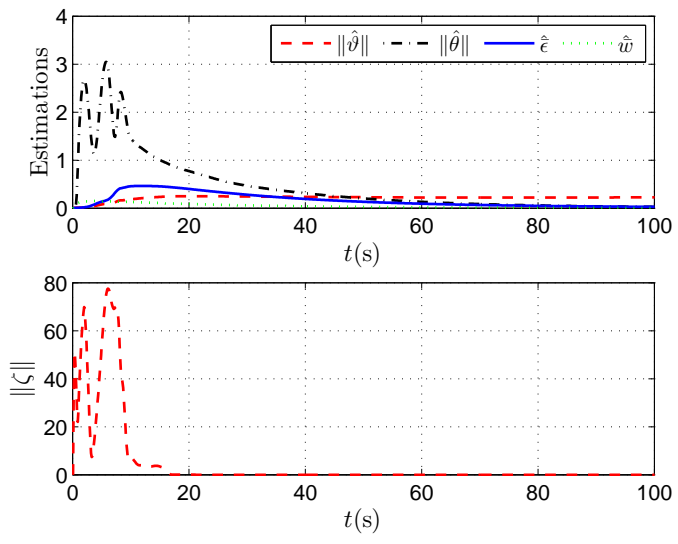

Fig. 6. Estimations and compensator states by (19).

$\boldsymbol{\delta}=\boldsymbol{\delta}_{0}+0.01 \operatorname{rand}(3,1)(\mathrm{Nm}), \boldsymbol{w}_{t}=100 \boldsymbol{w}_{0}+0.1 \operatorname{rand}(3,1)(\mathrm{N})$ and $\boldsymbol{\delta}_{t}=100 \boldsymbol{\delta}_{0}+0.1 \operatorname{rand}(3,1)(\mathrm{Nm})$, where

$$
\begin{aligned}
\boldsymbol{w}_{0} & =0.06\left[\sin \left(\frac{\pi}{40} t\right), \cos \left(\frac{\pi}{40} t\right), \sin \left(\frac{\pi}{45} t\right)\right]^{T}(\mathrm{~N}), \\
\boldsymbol{\delta}_{0} & =0.02\left[\sin \left(\frac{\pi}{45} t\right), \cos \left(\frac{\pi}{45} t\right), \sin \left(\frac{\pi}{40} t\right)\right]^{T}(\mathrm{Nm}) .
\end{aligned}
$$

In particular, the state uncertainties are also considered here as $\boldsymbol{p}_{e}(k+1)=\boldsymbol{p}_{e}(k)+0.001 \operatorname{rand}(6,1)$ and $\boldsymbol{q}_{e}(k+1)=$ $\boldsymbol{q}_{e}(k)+0.001 \operatorname{rand}(6,1)$ when the states are updated in the simulation, where $k$ denotes the numbers of sampling steps. The controller parameters are still set to the same values as previous ones. Then the numerical results are presented in Figures 7-10. From Figures 7-8, one can see that the convergence of relative pose and relative velocity still can be guaranteed with the acceptable response performance as ultimately uniformly bounded convergence of the full states and the non-violation of input and output constraints. Meanwhile, the relative pose outputs are always constrained in the prescribed values, and the control inputs are also constrained from Figures 7 and 9. Moreover, the online estimations and states of the compensator still bounded in Figure 10. These simulation results imply that the proposed control approach has stronger robustness in spite of the large uncertainties. 

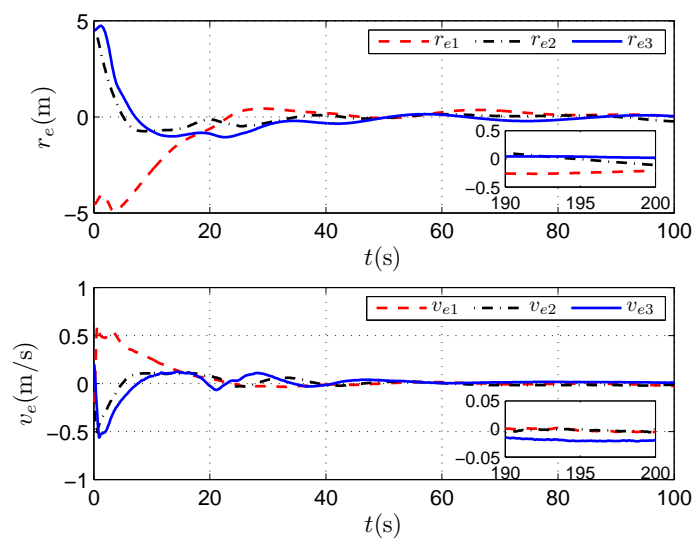

Fig. 7. Relative position and velocity under large uncertainties.
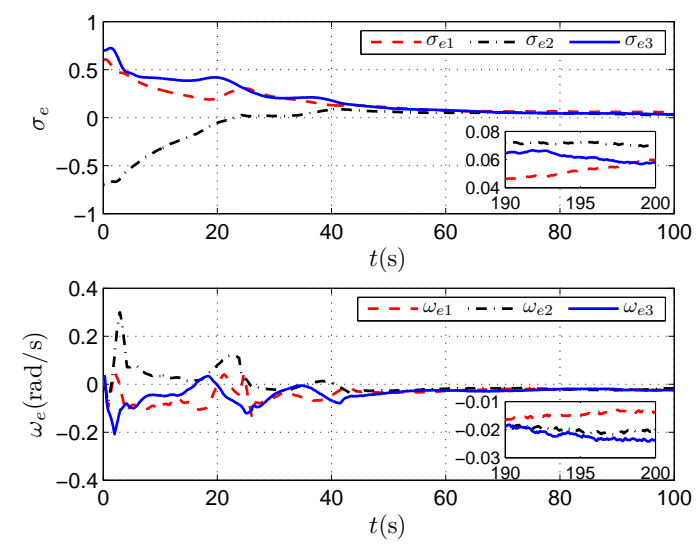

Fig. 8. Relative attitude and angular velocity under large uncertainties.
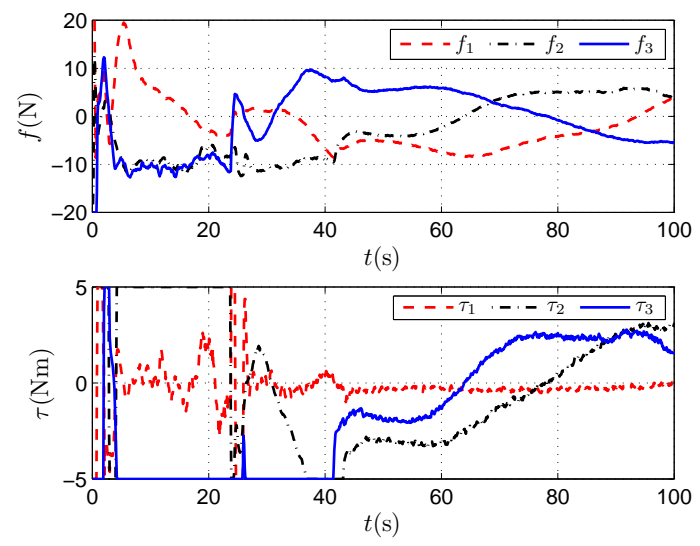

Fig. 9. Control forces and torques under large uncertainties.

\section{Performance Comparisons}

To show the advantages of the proposed control approach, the norm-wise adaptive backstepping control approach is employed. The reasons of choosing the norm-wise adaptive backstepping control approach are as follows. Firstly, the model in the relative dynamics has complicated couplings between unknown parameters of the target and the uncertain parameters
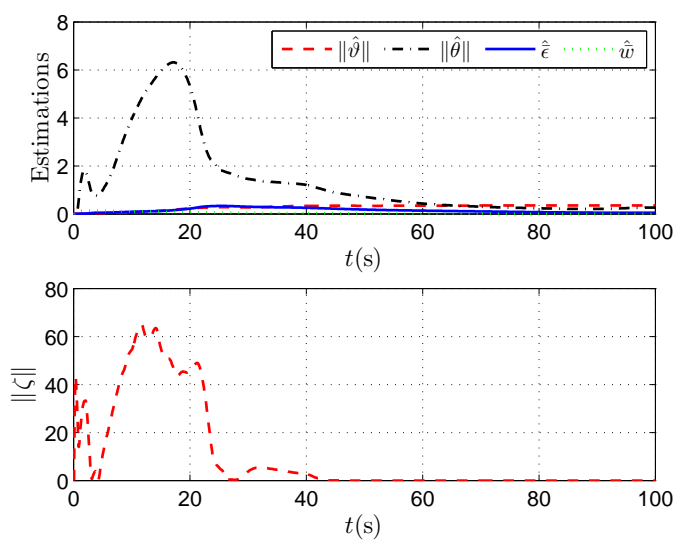

Fig. 10. Estimations and compensator states under large uncertainties.

of the chaser by multiplying state-dependent matrices, thus calculating the norm of the coupling terms in the model to extract the unknown constant parameters in the model is very convenient for designing the adaptive laws. Secondly, the norm-wise parameter adaptive law has less amount of the online estimations comparing with the traditional elementwise parameter adaptive laws, so that the computational burden of the controller can be decreased. Thirdly, the normwise adaptive control method can be easily combined with the variable structure control technique to design the simple robust adaptive control term for suppressing the unknown bounded disturbances in the model. Thus the performance of the controller is suitable for the comparisons with respect to the proposed controller in this study. The norm-wise adaptive backstepping controller is

$$
\begin{aligned}
\boldsymbol{u}_{0}= & -\sum_{i=1}^{6} \frac{\mathcal{B}_{e}^{T} E_{i}^{T} \boldsymbol{z}_{1}}{\overline{\boldsymbol{p}}_{e}^{T} E_{i} \overline{\boldsymbol{p}}_{e}-\boldsymbol{z}_{1}^{T} E_{i} \boldsymbol{z}_{1}}-K_{2} \boldsymbol{z}_{2}-K_{3} \boldsymbol{\zeta} \\
& -\boldsymbol{h}_{a}-\hat{\bar{\theta}}\|Y\| \tanh \left(\nu \boldsymbol{z}_{2}\right)-\hat{\bar{w}} \tanh \left(\nu \boldsymbol{z}_{2}\right)
\end{aligned}
$$

with adaptive laws and anti-windup compensator as

$$
\left\{\begin{array}{l}
\dot{\hat{\theta}}=-a_{1} a_{2} \hat{\bar{\theta}}+a_{2}\|Y\| \boldsymbol{z}_{2}^{T} \tanh \left(\nu \boldsymbol{z}_{2}\right) \\
\dot{\bar{w}}=-a_{3} a_{4} \hat{\bar{w}}+a_{4} \boldsymbol{z}_{2}^{T} \tanh \left(\nu \boldsymbol{z}_{2}\right) \\
\dot{\boldsymbol{\zeta}}=-\Pi \boldsymbol{\zeta}+\boldsymbol{u}_{\Delta}
\end{array}\right.
$$

where $K_{1}=0.1 I_{6}, K_{2}=\operatorname{diag}\left\{45 I_{3}, 250 I_{3}\right\}, K_{3}=2 I_{6}$, $\Pi=5 I_{6}, a_{1}=a_{2}=a_{3}=a_{4}=0.2, \nu=5$. In order to show quantitatively the advantages of the proposed controller, the integral of square error, the integrated absolute error, and the integrated time absolute error are used to evaluate the transient performance and steady-state performance for the controllers, where the definitions of these indexes are respectively as $I S E=\sqrt{\int_{0}^{T}\|\boldsymbol{u}(t)\|^{2} \mathrm{~d} t}, I A E=\sqrt{\sum_{i=1}^{6} \int_{0}^{T}\left|p_{e i}(t)\right| \mathrm{d} t}$, and $I T A E=\sqrt{\sum_{i=1}^{6} \int_{0}^{T} t\left|p_{e i}(t)\right| \mathrm{d} t}$. Under the simulation time $T=200(\mathrm{~s})$, it is derived easily that these indexes for the proposed controller (19) are respectively as $I S E_{1}=173.7380$, $I A E_{1}=14.4768$, and $I T A E_{1}=4.5780$, while the index values for the norm-wise adaptive backstepping control (25) are respectively as $I S E_{2}=236.0214, I A E_{2}=24.1340$, and $I T A E_{2}=7.6319$. Obviously, comparing these index values, 
the proposed controller (19) is better than the controller (25) in the view of the better response performance and smaller control effort. On the one hand, it is obviously seen from Figures 11-14 that the controller ensures the stability of the closed-loop system with the bounded signals and constrained inputs and outputs. On the other hand, in spite of the less computational burden of the norm-wise adaptive backstepping controller (25), it can also be seen that the proposed controller (19) has better transient and steady-state response performance with shorter time and higher precision by comparing Figures 3-6 and Figures 11-14.
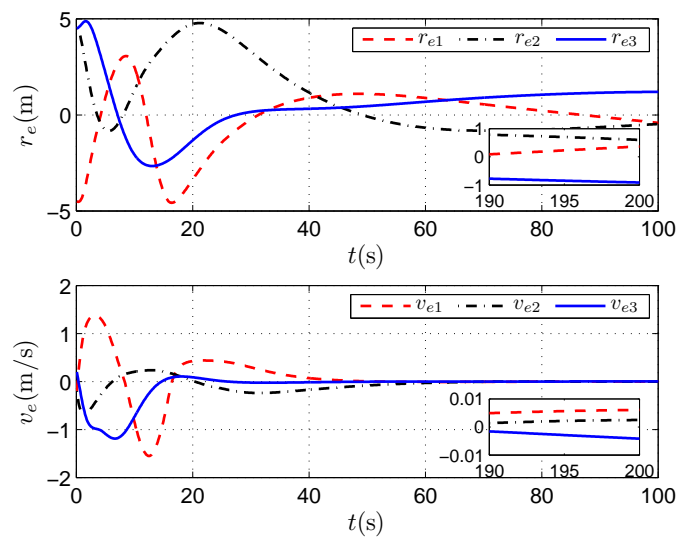

Fig. 11. Relative position and velocity by controller (25).
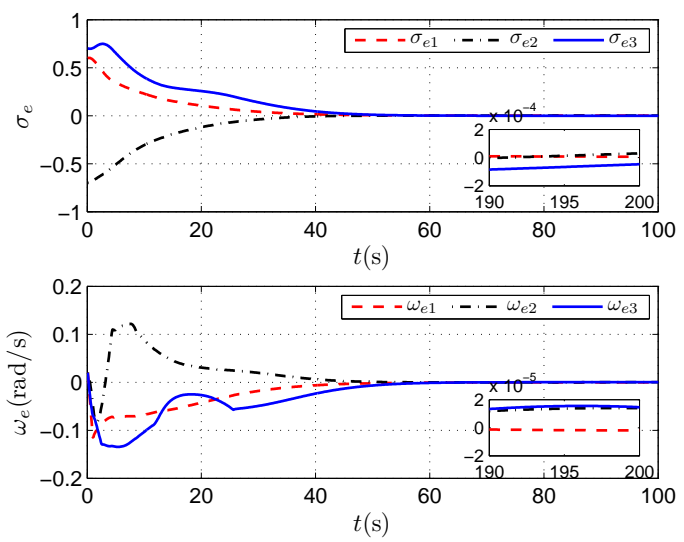

Fig. 12. Relative attitude and angular velocity by controller (25).

\section{CONCLUSIONS}

Based on the relative pose dynamics between feature points of two rigid bodies, the saturated intelligent adaptive pose coordination controller is developed by combining the adaptive hierarchical fuzzy logic system and robust adaptive backstepping technique. The proposed controller can guarantee the output constraints under the saturated control inputs, while the model uncertainties and unknown couplings are compensated by the adaptive fuzzy logic system. The transient and steadystate response performances can be improved by employing a smooth switching function-based hierarchical direct adaptive
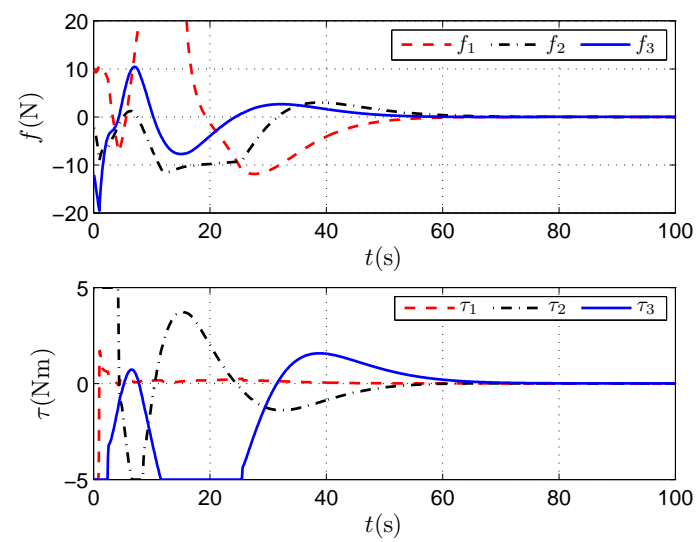

Fig. 13. Control forces and torques by controller (25).
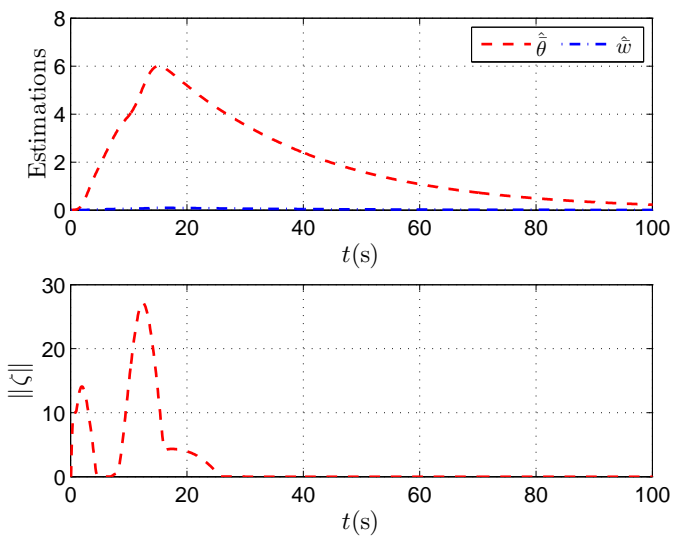

Fig. 14. Estimations and compensator states by controller (25).

fuzzy control approach, and the computational burden of the controller can be alleviated with hierarchical structure of the fuzzy logic systems. The rigorous stability analysis is shown in the Lyapunov framework, and it is concluded from theoretical and numerical results that the relative motion states ultimately converge to small neighborhoods of zero and the input and output constraints of the system can be guaranteed. The proposed modelling and control methodology can be used to guide many practical engineering missions, such as spacecraft docking, underwater docking, air refueling and shipboard landing. Future work will focus on improving the response time of the system under the same constraints by employing the finite/fixed-time control strategies.

\section{APPENDIX}

\section{A. Proof of the Theorem 1}

1) To prove $\hat{\boldsymbol{\vartheta}}_{k, j} \in \Omega_{\vartheta} \triangleq\left\{\hat{\boldsymbol{\vartheta}}_{k, j}:\left\|\hat{\boldsymbol{\vartheta}}_{k, j}\right\| \leq \Theta_{k, j}\right\}$, let $v_{k, j}=\frac{1}{2} \hat{\boldsymbol{\vartheta}}_{k, j}^{T,} \hat{\boldsymbol{\vartheta}}_{k, j}$. If the first sub-equation of (24) is satisfied, i.e. $\left\|\hat{\boldsymbol{\vartheta}}_{k, j}\right\|<\Theta_{k, j}$ or $\left\|\hat{\boldsymbol{\vartheta}}_{k, j}\right\|=\Theta_{k, j}$ and $z_{2 k} \hat{\boldsymbol{\vartheta}}_{k, j}^{T} \phi_{k, j} \leq 0$, then one has $\dot{v}_{k, j}=\kappa_{\rho} z_{2 k} \hat{\boldsymbol{\vartheta}}_{k, j}^{T} \phi_{k, j} \leq 0$, thus $\left\|\hat{\boldsymbol{\vartheta}}_{k, j}\right\| \leq \Theta_{k, j}$. If the second sub-equation of (24) is satisfied, i.e. $\left\|\hat{\boldsymbol{\vartheta}}_{k, j}\right\|=\Theta_{k, j}$ and $z_{2 k} \hat{\boldsymbol{\vartheta}}_{k, j}^{T} \boldsymbol{\phi}_{i, j}>0$, then one has $\dot{v}_{k, j}=\kappa_{\rho} z_{2 k} \hat{\boldsymbol{\vartheta}}_{k, j}^{T} \boldsymbol{\phi}_{k, j}-\kappa_{\rho} z_{2 k} \frac{\hat{\boldsymbol{\vartheta}}_{k, j}^{T} \hat{\boldsymbol{\vartheta}}_{k, j} \hat{\vartheta}_{k, j}^{T} \boldsymbol{\phi}_{k, j}}{\left\|\hat{\boldsymbol{\vartheta}}_{k, j}\right\|^{2}} \equiv 0$, thus 
$\left\|\hat{\boldsymbol{\vartheta}}_{k, j}\right\| \leq \Theta_{k, j}$. Therefore, the estimations $\hat{\boldsymbol{\vartheta}}_{k, j}$ for fuzzy logic systems are always bounded.

2) Define the optimal approximation of lumped disturbance in (17) is $\boldsymbol{w}^{*}=\left[w_{1}^{*}, \cdots, w_{6}^{*}\right]^{T}=$ $\left[\boldsymbol{\vartheta}_{1,17}^{* T} \boldsymbol{\phi}_{1,17}\left(y_{1,16}, s_{18}\right), \cdots, \boldsymbol{\vartheta}_{6,17}^{* T} \boldsymbol{\phi}_{6,17}\left(y_{6,16}, s_{18}\right)\right]^{T} \quad$ with the optimal parameter vectors $\boldsymbol{\vartheta}_{k}^{*}=\left[\boldsymbol{\vartheta}_{k, 1}^{* T}, \cdots, \boldsymbol{\vartheta}_{k, 17}^{* T}\right]^{T}=$ arg $\min _{\vartheta^{*} \in \Omega_{\vartheta}}\left\{\sup _{\boldsymbol{s} \in \Omega_{s}}\left|w_{k}-w_{k}^{*}\right|\right\}(k=1,2, \cdots, 6)$, where $\Omega_{s} \triangleq\left\{s:\|s\| \leq b_{1}\right\}$ is a bounded set determined by the function $\rho(\|s\|)$. Then the closed-loop dynamics can be derived by

$$
\begin{aligned}
\mathcal{M}_{e} \dot{\boldsymbol{z}}_{2}= & -\sum_{i=1}^{6} \frac{\mathcal{B}_{e}^{T} E_{i}^{T} \boldsymbol{z}_{1}}{\overline{\boldsymbol{p}}_{e}^{T} E_{i} \overline{\boldsymbol{p}}_{e}-\boldsymbol{z}_{1}^{T} E_{i} \boldsymbol{z}_{1}}-K_{2} \boldsymbol{z}_{2}-K_{3} \boldsymbol{\zeta} \\
& -Y \tilde{\boldsymbol{\theta}}+(1-\rho) \boldsymbol{\eta}_{n}+\rho \boldsymbol{\eta}_{t}+\boldsymbol{u}_{\Delta}+\boldsymbol{w}^{*}+\boldsymbol{\epsilon}
\end{aligned}
$$

where $\tilde{\boldsymbol{\theta}}=\hat{\boldsymbol{\theta}}-\boldsymbol{\theta}$ denotes the estimation error.

Define a candidate barrier Lyapunov function $v_{2}$ for the relative dynamics as

$$
\begin{aligned}
v_{2}= & v_{1}+\frac{1}{2}\left(\boldsymbol{z}_{2}^{T} \mathcal{M}_{\boldsymbol{e}} \boldsymbol{z}_{2}+\boldsymbol{\zeta}^{T} \boldsymbol{\zeta}\right)+\frac{1}{2 c_{2}} \tilde{\boldsymbol{\theta}}^{T} \tilde{\boldsymbol{\theta}} \\
& +\frac{1}{2 c_{4}} \tilde{\bar{\epsilon}}^{2}+\frac{1}{2 c_{6}} \tilde{\bar{w}}^{2}+\sum_{k=1}^{6} \sum_{j=1}^{17} \frac{\tilde{\boldsymbol{\vartheta}}_{k, j}^{T} \tilde{\boldsymbol{\vartheta}}_{k, j}}{2 \kappa}
\end{aligned}
$$

where $\tilde{\boldsymbol{\vartheta}}_{k, j}=\hat{\boldsymbol{\vartheta}}_{k, j}-\boldsymbol{\vartheta}_{k, j}^{*}$ is the estimation error. Then the time derivative of $v_{2}$ along the trajectory of the closed-loop system is

$$
\begin{aligned}
\dot{v}_{2}= & -\sum_{i=1}^{6} \frac{\boldsymbol{z}_{1}^{T} E_{i} K_{1} \boldsymbol{z}_{1}}{\overline{\boldsymbol{p}}_{e} E_{i} \overline{\boldsymbol{p}}_{e}-\boldsymbol{z}_{1}^{T} E_{i} \boldsymbol{z}_{1}}-\boldsymbol{z}_{2}^{T} K_{2} \boldsymbol{z}_{2}-\boldsymbol{z}_{2}^{T} K_{3} \boldsymbol{\zeta} \\
& +(1-\rho) \boldsymbol{z}_{2}^{T}\left[\boldsymbol{w}^{*}-\hat{\boldsymbol{w}}-(\bar{\epsilon}+\tilde{\bar{\epsilon}}) \tanh \left(\nu \boldsymbol{z}_{2}\right)+\boldsymbol{\epsilon}\right] \\
& +\rho \boldsymbol{z}_{2}^{T}\left[\boldsymbol{w}-(\bar{w}+\tilde{\bar{w}}) \tanh \left(\nu \boldsymbol{z}_{2}\right)\right]-\boldsymbol{z}_{2}^{T} Y \tilde{\boldsymbol{\theta}} \\
& +\boldsymbol{z}_{2}^{T} \boldsymbol{u}_{\Delta}-\boldsymbol{\zeta}^{T} \Pi \boldsymbol{\zeta}+\boldsymbol{\zeta}^{T} \boldsymbol{u}_{\Delta}+\frac{1}{c_{2}} \tilde{\boldsymbol{\theta}}^{T} \dot{\hat{\boldsymbol{\theta}}}+\frac{1}{c_{4}} \tilde{\bar{\epsilon}} \dot{\hat{\epsilon}} \\
& +\frac{1}{c_{6}} \tilde{\bar{w}} \dot{\overline{\hat{w}}}+\sum_{k=1}^{6} \sum_{j=1}^{17} \frac{1}{\kappa} \tilde{\boldsymbol{\vartheta}}_{k, j}^{T} \dot{\hat{\vartheta}}_{k, j}
\end{aligned}
$$

where $\tilde{\bar{\epsilon}}=\hat{\bar{\epsilon}}-\bar{\epsilon}$ and $\tilde{\bar{w}}=\hat{\bar{w}}-\bar{w}$ are estimation errors.

Furthermore, consider the following facts

$$
\begin{gathered}
\boldsymbol{z}_{2}^{T} \boldsymbol{\epsilon} \leq \sum_{k=1}^{6}\left|z_{2 k}\right| \bar{\epsilon} ; \quad \boldsymbol{z}_{2}^{T} \boldsymbol{w} \leq \sum_{k=1}^{6}\left|z_{2 k}\right| \bar{w} ; \\
\boldsymbol{z}_{2}^{T}\left(\boldsymbol{w}^{*}-\hat{\boldsymbol{w}}\right)=-\sum_{k=1}^{6} \sum_{j=1}^{17} z_{2 k} \tilde{\boldsymbol{\vartheta}}_{k, j}^{T} \boldsymbol{\phi}_{k, j} ; \\
\sum_{k=1}^{6}\left|z_{2 k}\right|-z_{2 k} \tanh \left(\nu z_{2 k}\right) \leq \frac{6 \iota}{\nu}, \iota=0.2785 ; \\
\tilde{\boldsymbol{x}}^{T} \hat{\boldsymbol{x}}=\tilde{\boldsymbol{x}}^{T}(\tilde{\boldsymbol{x}}+\boldsymbol{x})=\frac{1}{2}\left(\|\tilde{\boldsymbol{x}}\|^{2}+\|\hat{\boldsymbol{x}}\|^{2}-\|\boldsymbol{x}\|^{2}\right), \boldsymbol{x} \in \mathbb{R}^{n} ; \\
\sum_{k=1}^{6} \log \frac{\bar{p}_{e k}^{2}}{\bar{p}_{e k}^{2}-z_{1 k}^{2}} \leq \sum_{k=1}^{6} \frac{z_{1 k}^{2}}{\bar{p}_{e k}^{2}-z_{1 k}^{2}}
\end{gathered}
$$

for any $\bar{p}_{e k}>0$ and $\left|z_{1 k}(0)\right|<\bar{p}_{e k} ; 0 \leq \rho \leq 1$; and $3 \bar{\lambda}_{K_{3}}^{2}<$ $2 \underline{\lambda}_{K_{2}} \underline{\lambda}_{\Pi}$. Substituting the adaptive laws (20) and (24) into (28) yields

$$
\begin{aligned}
\dot{v}_{2} \leq & -\sum_{k=1}^{6} \underline{\lambda}_{K_{1}} \frac{z_{1 k}^{2}}{\bar{p}_{e k}^{2}-z_{1 k}^{2}}-\frac{1}{3} \underline{\lambda}_{K_{2}}\left\|\boldsymbol{z}_{2}\right\|^{2}-\frac{1}{2} \underline{\lambda}_{\Pi}\|\boldsymbol{\zeta}\|^{2}-\frac{c_{1}}{2}\|\tilde{\boldsymbol{\theta}}\|^{2} \\
& -\frac{c_{3}}{2}|\tilde{\bar{\epsilon}}|^{2}-\frac{c_{5}}{2}|\tilde{\bar{w}}|^{2}-\frac{1}{3 \underline{\lambda}_{K_{2}}}\left[\left\|\boldsymbol{z}_{2}\right\|-\frac{3 \bar{\lambda}_{K_{3}}^{2}}{2 \underline{\lambda}_{K_{2}}}\|\boldsymbol{\zeta}\|\right]^{2} \\
& -\frac{1}{2}\left[\underline{\lambda}_{\Pi}-\frac{3 \bar{\lambda}_{K_{3}}^{2}}{2 \underline{\lambda}_{K_{2}}}\right]\|\boldsymbol{\zeta}\|^{2}-\frac{1}{2 \underline{\lambda}_{\Pi}}\left[\|\boldsymbol{\zeta}\|-\frac{\left\|\boldsymbol{u}_{\Delta}\right\|}{\underline{\lambda_{\Pi}}}\right]^{2} \\
& -\frac{\left[\left\|\boldsymbol{z}_{2}\right\|-\frac{3\left\|\boldsymbol{u}_{\Delta}\right\|}{2 \underline{\lambda}_{K_{2}}}\right]^{2}}{3 \underline{\lambda}_{K_{2}}}-\frac{c_{1}}{2}\|\hat{\boldsymbol{\theta}}\|^{2}-\frac{c_{3}}{2}|\hat{\bar{\epsilon}}|^{2}-\frac{c_{5}}{2}|\hat{\bar{w}}|^{2}+\varpi \\
\leq & -\sum_{k=1}^{6} \underline{\lambda}_{K_{1}} \log _{\bar{p}_{e k}^{2}-z_{1 k}^{2}}-\frac{1}{3} \underline{\lambda}_{K_{2}}\left\|\boldsymbol{z}_{2}\right\|^{2}-\frac{1}{2} \underline{\lambda}_{\Pi}\|\boldsymbol{\zeta}\|^{2} \\
& -\frac{c_{1}}{2}\|\tilde{\boldsymbol{\theta}}\|^{2}-\frac{c_{3}}{2}|\tilde{\bar{\epsilon}}|^{2}-\frac{c_{5}}{2}|\tilde{\bar{w}}|^{2}+\varpi
\end{aligned}
$$

where $\varpi \triangleq \frac{3 \bar{u}_{\Delta}^{2}}{4 \underline{\lambda}_{K_{2}}}+\frac{\bar{u}_{\Delta}^{2}}{2 \underline{\lambda}_{\Pi}}+\frac{6 \iota(1-\rho) \bar{\epsilon}}{\nu}+\frac{6 \iota \rho \bar{w}}{\nu}+\frac{c_{1}}{2}\|\boldsymbol{\theta}\|^{2}+\frac{c_{3}}{2}|\bar{\epsilon}|^{2}+$ $\frac{c_{5}}{2}|\bar{w}|^{2}$. Furthermore, since $\left\|\hat{\boldsymbol{\vartheta}}_{k, j}\right\| \leq \Theta_{k, j},\left\|\boldsymbol{\vartheta}_{k, j}^{*}\right\| \leq \Theta_{k, j}$, then $\sum_{k=1}^{6} \sum_{j=1}^{17} \frac{1}{2 \kappa} \tilde{\boldsymbol{\vartheta}}_{k, j}^{T} \tilde{\boldsymbol{\vartheta}}_{k, j} \leq \sum_{k=1}^{6} \sum_{j=1}^{17} \frac{2 \Theta_{k, j}^{2}}{\kappa} \triangleq \varpi_{\Theta}$. Then, from the definition of $v_{2}$, one has

$$
\dot{v}_{2} \leq-a_{s}\left\|\boldsymbol{z}_{s}\right\|^{2}+\varpi \leq-a_{s} v_{2}+\varpi_{s}
$$

where $a_{s} \triangleq \min \left\{2 \underline{\lambda}_{K_{1}}, \frac{2 \underline{\lambda}_{K_{2}}}{3 \underline{\lambda}_{\mathcal{M}_{e}}}, \underline{\lambda}_{\Pi}, \frac{c_{2}}{c_{1}}, \frac{c_{4}}{c_{3}}, \frac{c_{6}}{c_{5}}\right\}, \quad \boldsymbol{z}_{s} \triangleq$ $\left[\sqrt{\log \frac{\bar{p}_{e 1}^{2}}{\bar{p}_{e 1}^{2}-z_{11}^{2}}}, \sqrt{\log \frac{\bar{p}_{e 2}^{2}}{\bar{p}_{e 2}^{2}-z_{12}^{2}}}, \sqrt{\log \frac{\bar{p}_{e 3}^{2}}{\bar{p}_{e 3}^{2}-z_{13}^{2}}}, \sqrt{\log \frac{\bar{p}_{e 4}^{2}}{\bar{p}_{e 4}^{2}-z_{14}^{2}}}\right.$, $\sqrt{\log \frac{\bar{p}_{e 5}^{2}}{\bar{p}_{e 5}^{2}-z_{15}^{2}}}, \sqrt{\log \frac{\bar{p}_{e 6}^{2}}{\bar{p}_{e 6}^{2}-z_{16}^{2}}},\left\|\boldsymbol{z}_{2}\right\|,\|\boldsymbol{\zeta}\|,\|\tilde{\boldsymbol{\theta}}\|,|\tilde{\bar{\epsilon}}|,|\tilde{\bar{w}}|^{T}, \quad$ and $\varpi_{s}=a_{s} \varpi_{\Theta}+\varpi$. Therefore, $v_{2}(t)$ is bounded by integrating both sides of (29) as

$$
v_{2}(t) \leq\left[v_{2}(0)-\frac{\varpi_{s}}{a_{s}}\right] \mathrm{e}^{-a_{s} t}+\frac{\varpi_{s}}{a_{s}} \leq v_{2}(0)+\frac{\varpi_{s}}{a_{s}}
$$

This implies that all signals in the closed-loop systems are always bounded. Meanwhile, since

$$
\frac{1}{2} \frac{\bar{p}_{e k}^{2}}{\bar{p}_{e k}^{2}-z_{1 k}^{2}} \leq \frac{1}{2} \sum_{k=1}^{6} \frac{\bar{p}_{e k}^{2}}{\bar{p}_{e k}^{2}-z_{1 k}^{2}} \leq v_{2}(0)+\frac{\varpi_{s}}{a_{s}}
$$

then taking exponentials on both sides of it yields

$$
\left|p_{e k}(t)\right|=\left|z_{1 k}(t)\right| \leq \bar{p}_{e k} \sqrt{1-\mathrm{e}^{-2\left[v_{2}(0)-\frac{w_{s}}{a_{s}}\right] \exp \left(-a_{s} t\right)+\frac{w_{s}}{a_{s}}}}
$$

Thus, if the initial constraints $\left|p_{e k}(0)\right|<\bar{p}_{e k}(k=1,2, \cdots, 6)$ are satisfied then the constraints of the system outputs $p_{e k}(t)$ are never violated, and $\lim _{t \rightarrow \infty}\left|z_{1 k}(t)\right| \leq \bar{p}_{e k} \sqrt{1-\mathrm{e}^{-2 \frac{\varpi}{a_{s}}}}$. In particular, if $\left\|\boldsymbol{z}_{s}\right\| \geq \frac{\varpi}{a_{s}}$ then $\dot{v}_{2} \leq 0$ from (29). Thus, it can be concluded that $\lim _{t \rightarrow \infty}\left\|\boldsymbol{z}_{s}(t)\right\| \leq \frac{\varpi}{a_{s}}, \lim _{t \rightarrow \infty}\left\|\boldsymbol{z}_{2}(t)\right\| \leq \frac{\varpi}{a_{s}}$, $\lim _{t \rightarrow \infty}\|\boldsymbol{\zeta}(t)\| \leq \frac{\varpi}{a_{s}}, \lim _{t \rightarrow \infty}\|\tilde{\boldsymbol{\theta}}(t)\| \leq \frac{\varpi}{a_{s}}, \lim _{t \rightarrow \infty}|\tilde{\bar{\epsilon}}(t)| \leq$ $\frac{\varpi}{a_{s}}$, and $\lim _{t \rightarrow \infty}|\overline{\bar{w}}(t)| \leq \frac{\varpi}{a_{s}}$. Furthermore, since $\boldsymbol{q}_{e}=$ $\boldsymbol{z}_{2}-\mathcal{B}_{e}^{T} K_{1} \boldsymbol{z}_{1}$ and $\left\|\boldsymbol{z}_{1}\right\| \leq\left\|\overline{\boldsymbol{p}}_{e}\right\|$, then $\lim _{t \rightarrow \infty}\left\|\boldsymbol{q}_{e}(t)\right\| \leq$ $\frac{\varpi}{a_{s}}+\left\|\mathcal{B}_{e}^{T} K_{1}\right\|\left\|\overline{\boldsymbol{p}}_{e}\right\|$. Therefore, the system states $\boldsymbol{p}_{e}$ and $\boldsymbol{q}_{e}$ are ultimately uniformly bounded by setting the suitable parameters of the controller, and the input and output constraints are guaranteed based on the proposed control strategy. 


\section{REFERENCES}

[1] J. Tjonnas and T. A. Johansen, Stabilization of automotive vehicles using active steering and adaptive brake control allocation, IEEE Trans. Control Syst. Technol., vol. 18, no. 3, pp. 545-558, May 2010.

[2] J.-M. Kai, T. Hamel and C. Samson, A unified approach to fixed-wing aircraft path following guidance and control, Automatica, vol. 108, pp. 108491 , Oct. 2019

[3] Y. Wang and S. Xu, Stabilization of coupled orbit-attitude dynamics about an asteroid utilizing Hamiltonian structure, Astrodynamics, vol. 2, no. 1, 53-67, 2018.

[4] X. Xiang, C. Yu, L. Lapierre, J. Zhang and Q. Zhang, Survey on fuzzy-logic-based guidance and control of marine surface vehicles and underwater vehicles, Int. J. Fuzzy Syst., vol. 20, no. 2, pp. 572-586, Feb. 2018.

[5] J. Sun and Z. Geng, Formation tracking control for multiple rigid bodies on matrix Lie groups: A system decomposition approach, Int. J. Robust Nonlinear Control, vol. 27, no. 18, pp. 4188-4207, 2017.

[6] L. Chang and Y. Jia, Adaptive control of a hose and drogue system with input nonlinearities and partial state constraints, Int. J. Control Autom. Syst., vol. 17, no. 10, pp. 2508-2520, Oct. 2019.

[7] Y. Huang, M. Zhu, Z. Zheng and M. Feroskhanc, Fixed-time autonomous shipboard landing control of a helicopter with external disturbances, Aerosp. Sci. Technol., vol. 84, pp. 18-30, Jan. 2019.

[8] H. Gui and G. Vukovich, Dual-quaternion-based adaptive motion tracking of spacecraft with reduced control effort, Nonlinear Dyn., vol. 83, no. 1-2, 597-614, 2016.

[9] P. Batista, C. Silvestre and P. Oliveira, A two-step control approach for docking of autonomous underwater vehicles, Int. J. Robust Nonlinear Control, vol. 25, no. 10, pp. 1528-1547, Jul. 2015.

[10] B. Bischof, T. Glueck and A. Kugi, Combined path following and compliance control for fully actuated rigid body systems in 3-D space, IEEE Trans. Control Syst. Technol., vol. 25, no. 5, pp. 1750-1760, 2017.

[11] G. Di Mauro, M. Schlotterer, S. Theil and M. Lavagna, Nonlinear control for proximity operations based on differential algebra, J. Guid. Control Dyn., vol. 38, no. 11, pp. 2173-2187, 2015.

[12] R. W. Beard, J. Lawton and F. Y. Hadaegh, A coordination architecture for spacecraft formation control, IEEE Trans. Control Syst. Technol., vol. 9, no. 6, pp. 777-790, Nov. 2001.

[13] A. Astolfi and R. Ortega, Immersion and invariance: A new tool for stabilization and adaptive control of nonlinear systems, IEEE Trans. Autom. Control, vol. 48, no. 4, pp. 590-606, Apr. 2003.

[14] N. Hovakimyan, C. Cao, E. Kharisov, E. Xargay and I. M. Gregory, $L_{1}$ adaptive control for safety-critical systems guaranteed robustness with fast adaptation, IEEE Trans. Control Syst. Mag., vol. 233, no. 11, pp. 4129-4140, Sep. 2019.

[15] M. Sugeno, An introductory survey of fuzzy control, Info. Sci., vol. 36, no. $1-2$, pp. $59-83,1985$.

[16] G. V. S. Raju, J. Zhou and R. A. Kisner, Hierarchical fuzzy control, Int J. Control, vol. 54, no. 5, pp. 1201-1216, 1991.

[17] G.V. S. Raju and J. Zhou, Adaptive hierarchical fuzzy controller, IEEE Trans. Syst. Man Cyber., vol. 23, no. 4, pp. 973-331, Jul. 1993.

[18] L.-X. Wang, Adaptive Fuzzy Systems and Control: Design and Stability Analysis, Prentice Hall, 1994

[19] M. Sugeno and M. Nishida, Fuzzy control of model car, Fuzzy Sets Syst., vol. 16, no. 2, pp. 103-113, 1985.

[20] H.-M. Wu and M. Karkoub, Hierarchical fuzzy sliding-mode adaptive control for the trajectory tracking of differential-driven mobile robots, Int. J. Fuzzy Syst., vol. 21, no. 1, pp. 33-49, Feb. 2019.

[21] M. Sugeno, I. Hirano, S. Nakamura and S. Kotsu, Development of an intelligent unmanned helicopter, Proc. 1995 IEEE Int. Conf. Fuzzy Syst., Yokohama, Japan, IEEE Publishing House, pp. 33-34, 1995.

[22] L. Sun and W. Huo, Adaptive fuzzy control of spacecraft proximity operations using hierarchical fuzzy systems, IEEE-ASME Trans. Mechatron., vol. 21, no. 3, pp. 1629-1640, Jun. 2016.

[23] L. Sun, W. He and C. Sun, Adaptive fuzzy relative pose control of spacecraft during rendezvous and proximity maneuvers, IEEE Trans. Fuzzy Syst., vol. 26, no. 6, pp. 3440-3451, Dec. 2018.

[24] M. Mansouri, M. Teshnehlab and M. A. Shoorehdeli, Indirect adaptive hierarchical fuzzy sliding mode controller for a class of nonlinear systems, J. Intel. Fuzzy Syst., vol. 30, no. 3, pp. 1377-1390, 2016.

[25] K. P. Tee, S. S. Ge and E. H. Tay, Barrier Lyapunov functions for the control of output-constrained nonlinear systems, Automatica, vol. 45, pp. 918-927, 2009

[26] Y. Zhong, Globally stable adaptive system design for minimum phase SISO plants with input saturation, Automatica, vol. 41, no. 9, pp. 1539 1547,2005
[27] C. Wen, J. Zhou, Z. Liu and H. Su. Robust adaptive control of uncertain nonlinear systems in the presence of input saturation and external disturbance, IEEE Trans. Autom. Control, vol. 56, no. 7, pp. 1672-1678, 2011.

[28] L. Sun, Saturated adaptive output-constrained control of cooperative spacecraft rendezvous and docking, IEEE/CAA J. Autom. Sinica, vol. 6, no. 6, pp. 1462-1470, Jul. 2019.

[29] W. Shi, F. Yan and B. Li, Adaptive fuzzy decentralized control for a class of nonlinear systems with different performance constraints, Fuzzy Sets Syst., vol. 374, pp. 1-22, Nov. 2019.

[30] Z. Peng, J. Wang and Q.-L. Han, Path-following control of autonomous underwater vehicles subject to velocity and input constraints via neurodynamic optimization, IEEE Trans. Ind. Electron., vol. 66, no. 11, pp.8724-8732, Nov. 2019.

[31] F. Zhang and G. Duan, Manipulator-actuated adaptive integrated translational and rotational stabilization for spacecraft in proximity operations with control constraint, Int. J. Control Autom. Syst., vol. 16, no. 5, pp. 2103-2113, 2018.

[32] J. Jiang and A. Astolfi, State and output-feedback shared-control for a class of linear constrained systems, IEEE Trans. Autom. Control, vol. 61, no. 10 , pp. 3209-3214, Oct. 2016

[33] K. P. Tee and S. S. Ge, Control of nonlinear systems with partial state constraints using a barrier Lyapunov function, Int. J. Control, vol. 84, no. 12, pp. 2008-2023, 2011.

[34] H. Schaub and J. L. Junkins, Analytical Mechanics of Space Systems, Reston: AIAA, 2003.

[35] A. H. J. De Ruiter and C. J. Damaren, Effect of attitude parameterization on the perfermance of passivity-based adaptive attitude control, AIAA Guid., Nav. Control Conf. Exihib., Montreal, Canada, AIAA-2001-4154, 2001.

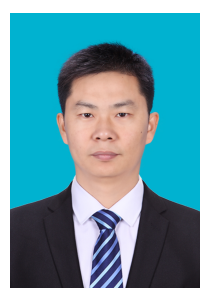

Liang Sun received the M.Sc. and Ph.D. degrees in control theory and control engineering from Beihang University, China, in 2011 and 2016, respectively. He was a Postdoctoral Fellow in mechanical engineering with Beihang University from 2015 to 2017, and a Visiting Scholar with Loughborough University, UK, from 2019 to 2020 . He joined University of Science and Technology Beijing as an Associate Professor in 2017. His current research interests include nonlinear mechanical systems control, aerospace control and intelligent control.

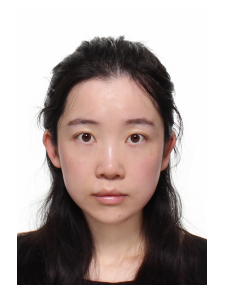

Jingjing Jiang received the B.E. Degree in Electrical and Electronic Engineering from the University of Birmingham, UK, and the Harbin Institute of Technology, China, in 2010, the M.Sc. degree in Control Systems from Imperial College London, UK, in 2011, and the Ph.D degree from Imperial College London, in 2016. She carried out research as part of the Control and Power Group at Imperial College and joined Loughborough University as a Lecturer in September 2018. Her current research interests include driver assistance control and autonomous vehicle control design, control design of systems with constraints and human-in-the-loop. 